TITLE:

\title{
Generalized Hough transform for the stress inversion of calcite twin data
}

$\operatorname{AUTHOR}(S)$ :

Yamaji, Atsushi

\section{CITATION:}

Yamaji, Atsushi. Generalized Hough transform for the stress inversion of calcite twin data. Journal of Structural Geology 2015, 80: 2-15

\section{ISSUE DATE:}

2015-11

URL:

http://hdl.handle.net/2433/203651

\section{RIGHT:}

(c) 2015. This manuscript version is made available under the CC-BY-NC-ND 4.0 license

http://creativecommons.org/licenses/by-nc-nd/4.0/; The full-text file will be made open to the public on 1 November 2017 in accordance with publisher's 'Terms and Conditions for Self-Archiving'.; This is not the published version. Please cite only the published version,; この論文は出版社版でありません。引用の際には出版社版をご確認ご利用ください。 


\title{
Generalized Hough transform for the stress inversion of calcite twin data
}

\author{
Atsushi Yamaji ${ }^{a, *}$ \\ ${ }^{a}$ Division of Earth and Planetary Sciences, Graduate School of Science, Kyoto University, Kyoto 606-8502, Japan
}

\begin{abstract}
Since the mechanical twinning along calcite $e$-planes has a critical resolved shear stress, not only principal stress axes but also differential stress can be determined from the orientations of twin lamellae. Based on the five-dimensional stress space that fulfills the principle of coordinate invariance, it is shown the inversion of twin and untwin data is comparable with fitting a spherical cap to data points on a unit sphere in the space. The principal stress orientations and stress ratio are indicated by the center of the cap, whereas differential stress is denoted by the size of the cap. Based on this geometrical interpretation, a new inversion scheme is proposed. The method is demonstrated to be robust to sampling bias, variability in the critical resolved shear stress, and the heterogeneity of data.
\end{abstract}

Keywords: stress space, inverse method, sampling bias, paleostress

\section{Introduction}

The stress inversion of calcite $e$-twin data has been used to elucidate paleostresses at the shallow levels of the crust. The inverse methods determine not only the principal stress axes but also stress ratio and differential stress from the orientations of twinned and untwinned $e$-planes (Etchecopar, 1984; Laurent et al., 1990). Principal stress orientations and differential stress magnitudes have been determined in various areas using $e$-twin data (e.g., Lacombe, 2010; Amrouch et al., 2010, 2011; Beaudoin et al., 2012).

Recently, Yamaji (2015) reformulated the theories of Takeshita et al. (1987) and Fry (2001) on the twinning condition to fulfill the principle of coordinate invariance: Physical laws must be formulated in mathematical forms that are independent from the choice of a coordinate system (e.g., Ottosen and Ristinmaa, 2005). In addition, Yamaji (2015) delineated the range of stresses constrained by a twin or untwin datum.

It is known in data mining that if the range of solutions constrained by a datum is defined, the generalized Hough transform (Ballard, 1981) can detect one or more solutions that explain a given dataset. Therefore, it is straightforward for the transform to detect stresses from heterogeneous calcite twin data. Here, we propose such an inversion scheme. The next section is devoted to explain the parts of the theory essential for introducing the inversion scheme in Section 3. Then, the method is demonstrated with synthetic data sets to be robust to sampling bias, the variability of the critical resolved shear stress, and the heterogeneity of data. Finally, the present scheme is compared with previous ones.

\footnotetext{
*Phone: +81 75753 4266; fax: +81 757534189 .

Email address: yamaji@kueps. kyoto-u.ac.jp (Atsushi Yamaji)
}

\section{Basic equations and inequalities}

The present method is based on the mathematical formulation of the accompanying paper (Yamaji, 2015), which was founded only upon the twinning condition, $\tau \geq \tau_{\mathrm{c}}$, where $\tau$ is the resolved shear stress along the twin gliding direction of an $e$-plane and $\tau_{\mathrm{c}}$ is its threshold. However, this critical value is known to depend on temperature at the time of twinning and grain size to some extent (Lacombe, 2010, and references therein). Accordingly, we use not only differential stress, $D$, but also non-dimensional differential stress, $\underline{D}=D / \tau_{\mathrm{c}}$, to circumvent the uncertainty of the critical value. That is, differential stresses are denoted by the multiples of $\tau_{\mathrm{c}}$. For example, when we deal with twin lamellae that were formed with $\tau_{\mathrm{c}}=10 \mathrm{MPa}$, the value, $\underline{D}=5$, indicates $D=50 \mathrm{MPa}$. In case the $\tau_{\mathrm{c}}$ value is uncertain, the $\underline{D}$ value can be determined from twin data.

\subsection{Sigma- and epsilon-vectors}

If the information of stress magnitude is abstracted away from stress tensor, the resultant quantity called reduced stress tensor carries only the information of principal axes and stress ratio, $\Phi=\left(\sigma_{2}-\sigma_{3}\right) /\left(\sigma_{1}-\sigma_{3}\right)$. Suppose that the unit vectors $\boldsymbol{q}^{(1)}, \boldsymbol{q}^{(2)}$ and $\boldsymbol{q}^{(3)}$ make a right-hand system in this order; and are parallel to the $\sigma_{1^{-}}, \sigma_{2-}$ and $\sigma_{3}$-axes, respectively. We use the reduced stress tensor of the form,

$$
\boldsymbol{\varsigma}=\frac{1}{3 \lambda} \boldsymbol{Q}\left(\begin{array}{ccc}
2-\Phi & 0 & 0 \\
0 & 2 \Phi-1 & 0 \\
0 & 0 & -\Phi-1
\end{array}\right) \boldsymbol{Q}^{\mathrm{T}},
$$

where $\boldsymbol{Q}$ is the orthogonal matrix whose ijth component is $q_{i}^{(j)}$, and

$$
\lambda=\sqrt{\left(\Phi^{2}-\Phi+1\right) / 3} .
$$

$\boldsymbol{S}$ is a deviatoric tensor, meaning that $\varsigma_{11}+\varsigma_{22}+\varsigma_{33}=0$. Deviatoric stress tensor is denoted by $\boldsymbol{T}=\lambda D \boldsymbol{S}$ (Yamaji, 2015). 
$\lambda$ has the minimum value $(1 / 2)$ at $\Phi=1 / 2$, and the maximal one $(1 / \sqrt{3} \approx 0.58)$ at $\Phi=0$ and 1 . Stress ratio simply indicates the shape of stress ellipsoid, whereas $\lambda$ relates differential stress to equivalent stress, $T_{\mathrm{E}}$, which is a scalar quantity representing the components of $\boldsymbol{T}$ to predict yielding under multiaxial stress conditions (Hill, 1950). The quantity is written as (Yamaji, 2007, Eqs. 4.15, 10.37)

$$
T_{\mathrm{E}}=\sqrt{3 T_{\mathrm{II}}}=\sqrt{3} \lambda \underline{D} \tau_{\mathrm{c}},
$$

where the quadratic quantity of the components of $\boldsymbol{T}$, i.e.,

$$
T_{\mathrm{II}}=\frac{1}{2}\left(T_{11}^{2}+T_{22}^{2}+T_{33}^{2}\right)+T_{12}^{2}+T_{23}^{2}+T_{31}^{2}
$$

is called the second basic invariant of $\boldsymbol{T}$. The values of the components themselves are affected by the choice of coordinate orientations, but that of $T_{\mathrm{II}}$ is invariant under coordinate rotations. The reduced stress tensor was defined for its second basic invariant to satisfy $\varsigma_{\mathrm{II}}=1$ : We defined the reduced stress tensor as Eq. (1) for its second basic invariant to fulfill this condition.

The present method searches for the deviatoric stress tensors in a five-dimensional parameter space that explain a dataset. Since $\boldsymbol{T}$ is a symmetric tensor satisfying $T_{11}+T_{22}+T_{33}=0, \boldsymbol{T}$ has five-degrees of freedom such that

$$
\boldsymbol{T}=\left(\begin{array}{ccc}
T_{11} & T_{12} & T_{13} \\
T_{12} & T_{22} & T_{23} \\
T_{13} & T_{23} & -T_{11}-T_{22}
\end{array}\right),
$$

where $\boldsymbol{T}$ is denoted only by its five components, $T_{11}, T_{12}, T_{13}$, $T_{22}$ and $T_{23}$. The five components can be used to define a fivedimensional parameter space to indicate $\boldsymbol{T}$, for example, by the Cartesian coordinates $\left(T_{11}, T_{12}, T_{13}, T_{22}, T_{23}\right)$. However, such a parameter space is known to be inappropriate, because it does not fulfill the principle of coordinate invariance. It means that the results of computation can be affected by the choice of coordinate orientations in the physical space (Sato and Yamaji, 2006a). Instead, we define the parameter space to have the coordinates,

$$
\left(\left(T_{11}-T_{33}\right) / 2,\left(-T_{11}+2 T_{22}-T_{33}\right) / 2 \sqrt{3}, T_{23}, T_{31}, T_{12}\right) .
$$

Let us define the five-dimensional position vector,

$$
\vec{x}=\lambda D \vec{\sigma},
$$

where

$$
\vec{\sigma}=\left(\left(\varsigma_{11}-\varsigma_{33}\right) / 2,\left(-\varsigma_{11}+2 \varsigma_{22}-\varsigma_{33}\right) / 2 \sqrt{3}, \varsigma_{23}, \varsigma_{31}, \varsigma_{12}\right) .
$$

By the definition of $\boldsymbol{\varsigma}$ (Eq. 1), $\vec{\sigma}$ is a unit vector; and it follows from Eqs. (4) and trace $\boldsymbol{T}=0$ that $|\vec{x}|^{2}=x_{1}^{2}+x_{2}^{2}+x_{3}^{2}+x_{4}^{2}+x_{5}^{2}=$ $T_{\mathrm{II}}$. It means that $|\vec{x}|$ is invariant under coordinate rotations. Since $\boldsymbol{T}$ is proportional to $\boldsymbol{s}$, the components of $\vec{x}$ are equivalent with Eq. (5). Let us define the non-dimensional version of $\vec{x}$ as $\underline{\vec{x}}=\vec{x} / \tau_{\mathrm{c}}$.

We call $\vec{\sigma}$ a sigma-vector. It should be noted that $\vec{\sigma}$ has a one-to-one correspondence with $\boldsymbol{\varsigma}$, allowing us to identify them

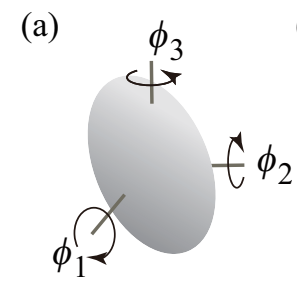

(b)

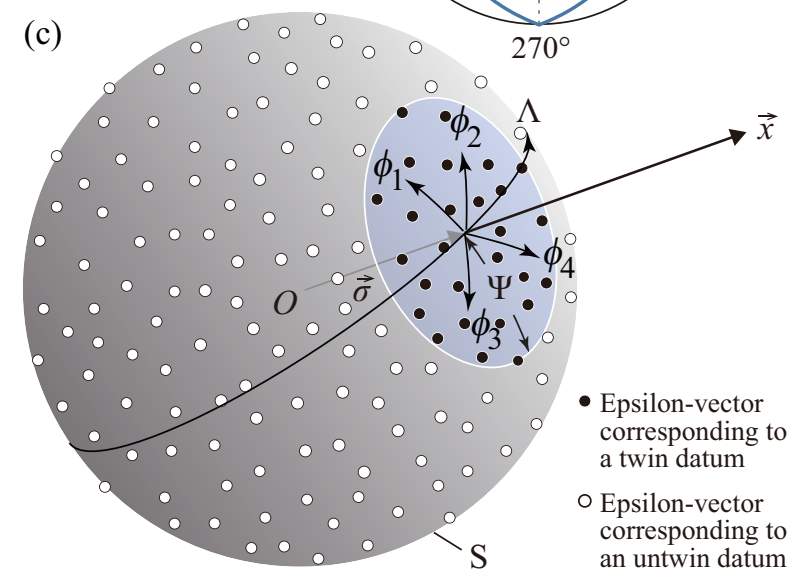

Figure 1: (a) Schematic illustration for a stress ellipsoid in the physical space, the attitude of which is denoted by rotation angles about the coordinate axes, $\phi_{1}$, $\phi_{2}$ and $\phi_{3}$, whereas the shape of the ellipsoid is denoted by $\Phi$ or $\Lambda$. (b) Diagram showing the correspondence between $\Phi$ and $\Lambda$. (c) Schematic illustration for a unit sphere, $S$, in a five-dimensional space and the five-dimensional position vector, $\vec{x}$ that represents $\boldsymbol{T}$. Epsilon-vectors corresponding to twin and untwin data are represented, respectively, by solid and open circles. The former vectors exist in the spherical cap with the center, $\vec{\sigma}$, and radius, $\Psi$, on $\mathrm{S}$. The curves defined by the parameters, $\Lambda$ and $\phi_{i}$, meet at a right angle on $\mathrm{S}$.

(Appendix A). It follows from $|\vec{\sigma}|=1$ that $\vec{\sigma}$ represents a point on a unit sphere, $\mathrm{S}$, in the five-dimensional space, which has the spherical coordinates, $\phi_{1}, \phi_{2}, \phi_{3}$ and $\Lambda$. The first three coordinates represent the attitude of stress ellipsoid in the physical space (Fig. 1). $\Lambda$ is called Lode angle in plasticity theory, and represents the shape of the ellipsoid. $\Phi$ is the function of $\Lambda$ with the periodicity of $120^{\circ}$ (Yamaji and Sato, 2006), i.e., $\Phi=2 \sin \ell(\Lambda) /[\sqrt{3} \cos \ell(\Lambda)+\sin \ell(\Lambda)]$, where $\ell(\Lambda)=\Lambda+30^{\circ}$ for $-30^{\circ} \leq \Lambda<30^{\circ}$, and $\ell(\Lambda)=90^{\circ}-\Lambda$ for $30^{\circ} \leq \Lambda<90^{\circ}$ (Fig. 1b).

Similar to the correspondence between $\boldsymbol{\varsigma}$ and $\vec{\sigma}$, twinning along an $e$-plane is represented by a unit vector, $\vec{\epsilon}$, in the fivedimensional space. This vector is obtained as follows. Let $\boldsymbol{e}$ and $\boldsymbol{g}$ be the unit vectors indicating the pole and gliding direction of the plane, respectively. Then, reduced strain tensor is defined as $\boldsymbol{\varepsilon}=\boldsymbol{g} \boldsymbol{e}^{\mathrm{T}}+\boldsymbol{e} \boldsymbol{g}^{\mathrm{T}}$, which carries only the information of the direction of strain by twinning, and strain magnitude is abstracted away from $\varepsilon$. The five-dimensional unit vector representing an $e$-plane is defined as

$$
\vec{\epsilon}=-\left(\left(\varepsilon_{11}-\varepsilon_{33}\right) / 2,2\left(-\varepsilon_{11}+2 \varepsilon_{22}-\varepsilon_{33}\right) / \sqrt{3}, \varepsilon_{23}, \varepsilon_{31}, \varepsilon_{12}\right),
$$

and is called an epsilon-vector. We identify this unit vector with a point on $S$. 


\subsection{Spherical cap}

Twinning occurs if the resolved shear stress, $\tau$, along the gliding direction exceeds a critical value, $\tau_{\mathrm{c}}$. Since the former satisfies the equation (Yamaji, 2015),

$$
\tau=\lambda D \vec{\sigma} \cdot \vec{\epsilon},
$$

It can be seen from Eq. (6) that the twinning condition, $\tau \geq \tau_{\mathrm{c}}$, is identical with $\vec{\epsilon} \cdot \vec{\sigma} \lambda D \geq \tau_{\mathrm{c}}$ and

$$
\vec{\epsilon} \cdot \vec{x} \geq \tau_{\mathrm{c}} .
$$

A twin or untwin datum denoted by $\vec{\epsilon}$ is said to be compatible with or explained by the deviatoric stress tensor presented by $\vec{x}$ or $\boldsymbol{T}$, if the inequality (10) is satisfied. An untwin datum denoted by $\vec{\epsilon}$ is said to be so, if the inequality,

$$
\vec{\epsilon} \cdot \vec{x}<\tau_{\mathrm{c}},
$$

is satisfied. It follows from Eq. (9) that these inequalities are combined into the expression,

$$
H\left( \pm \lambda D \vec{\sigma} \cdot \vec{\epsilon} \mp \tau_{\mathrm{c}}\right)=1
$$

where the upper and lower signs correspond, respectively, to the inequalities (9) and (11), and $H$ is the Heaviside step function,

$$
H(x)= \begin{cases}0 & (x<0) \\ 1 & (0 \leq x)\end{cases}
$$

The twinning condition has an important geometrical interpretation in relation to S (Yamaji, 2015). Since $\vec{\sigma}$ and $\vec{\epsilon}$ are unit vectors, we have $\cos \psi=\vec{\sigma} \cdot \vec{\epsilon}$, where $\psi$ is the angle made by the vectors. The condition, $\tau_{\mathrm{c}} \leq \tau$, can be rewritten in terms of Eq. (9) as $\lambda \underline{D} \cos \psi \geq 1$. It follows that $\vec{\epsilon}$ is on the spherical cap with the radius of

$$
\Psi=\cos ^{-1}(1 / \lambda \underline{D}),
$$

if the stress, $\underline{\vec{x}}=\lambda \underline{D} \vec{\sigma}$, explains the twin datum, $\vec{\epsilon}$ (Fig. 1c).

\section{Inverse method}

Based on the inequalities (10) and (11), we demonstrate that an inversion scheme can be formed to determine the deviatoric stress tensors compatible with given twin and untwin data. The inverse method aims at determining the optimal vector, $\vec{x}_{\mathrm{opt}}$, from a dataset. Once this vector is obtained, the vector is decomposed into the combination of optimal stress axes, stress ratio and differential stress (Appendix A).

Suppose that we have $N_{\mathrm{t}}$ twin and $N_{\mathrm{u}}$ untwin data. We refer $N$ to the total number of data: $N=N_{\mathrm{t}}+N_{\mathrm{u}}$. The data are represented by the epsilon-vectors, $\left\{\vec{\epsilon}_{\mathrm{t}}^{(1)}, \ldots, \vec{\epsilon}_{\mathrm{t}}^{\left(N_{\mathrm{t}}\right)}\right\}$ and $\left\{\vec{\epsilon}_{\mathrm{u}}^{(1)}, \ldots, \vec{\epsilon}_{\mathrm{u}}^{\left(N_{\mathrm{u}}\right)}\right\}$, respectively: The subscripts 't' and 'u' indicate the quantities of twin and untwin data.
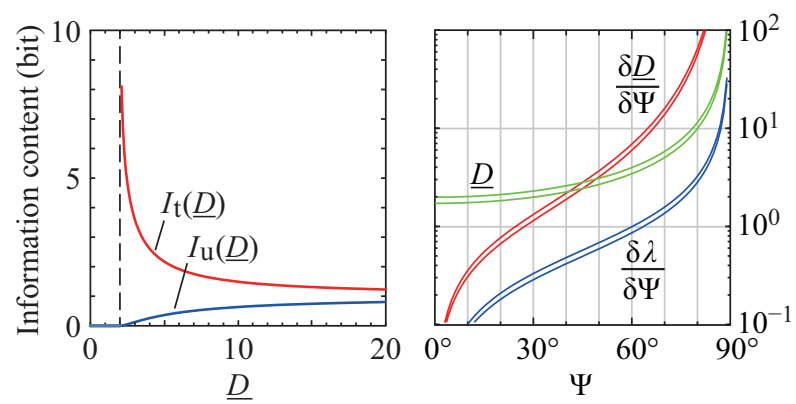

Figure 2: (a) Information contents of a twin datum and an untwin datum. (b) The coefficients of the error propagation from $\Psi$ to $\lambda$ and $D$ (Eqs. 19 and 20). $D$ is indicated in MPa, and was evaluated by Eq. (13). Double lines indicate the extreme cases with $\lambda=1 / 2$ and $1 / \sqrt{3}$, which correspond to $\Phi=1 / 2$ and $\Phi=1 / 2 \pm 1 / 2$, respectively.

\subsection{Inversion scheme}

Let us consider how $\underline{\underline{x}}$ is determined from the data. Once this vector is determined, $\vec{x}$ is obtained simply by multiplying $\underline{\vec{x}}$ with an appropriate $\tau_{\mathrm{c}}$ value; and $\boldsymbol{T}$ is obtained through the procedure in Appendix A.

In case the data set is homogeneous (in other words, all twin lamella were formed under the same stress state), $\underline{\vec{x}}$ satisfies the simultaneous inequalities,

$$
\begin{aligned}
& \vec{\epsilon}_{\mathrm{t}}^{(1)} \cdot \underline{\vec{x}} \geq 1, \ldots, \vec{\epsilon}_{\mathrm{t}}^{\left(N_{\mathrm{t}}\right)} \cdot \underline{\vec{x}} \geq 1 \\
& \vec{\epsilon}_{\mathrm{u}}^{(1)} \cdot \underline{\vec{x}}<1, \ldots, \vec{\epsilon}_{\mathrm{u}}^{\left(N_{\mathrm{u}}\right)} \cdot \underline{\vec{x}}<1 .
\end{aligned}
$$

That is, $\underline{\vec{x}}$ is the solution of this linear system (Laurent et al., 1981; Fry, 2001). The $i$ th inequality constrains the possible region for $\underline{\vec{x}}$ to one side of the hyperplane denoted by $\vec{\epsilon}^{(i)} \cdot \underline{\vec{x}}=1$. The solution of the system is called the feasible region, which is a polytope (a high dimensional analogue of a polyhedron) in the five-dimensional space. It is obvious from Fig. 1 that untwin data place the upper bound for the size of a spherical cap, which indicates differential stress.

An inversion scheme was devised in this work to detect stress(es) as follows. If a data set is homogeneous and free from disturbances coming from measurement errors, sampling bias, etc., the simultaneous inequalities (14 and 15) have a solution: A feasible region exists. However, disturbances can give rise to the non-existence of the solution. Even in this case, the number of the inequalities satisfied by an arbitrarily chosen $\underline{\vec{x}}$ is a measure to estimate the fitness of $\underline{\vec{x}}$ for a given data set. Let $n_{\mathrm{t}}(\underline{\vec{x}})$ and $n_{\mathrm{u}}(\underline{\vec{x}})$ be the numbers for the twin and untwin data, respectively. It would appear that a better solution has a larger the summation, $n_{\mathrm{t}}(\underline{\vec{x}})+n_{\mathrm{t}}(\underline{\vec{x}})$, but this is not true. It should be noted that the inequalities in (14) and (15) have different significance, because the constraint on $\boldsymbol{T}$ from a twin datum is always greater than that from an untwin datum (Yamaji, 2015). Their difference depends on the differential stress that should be read from data. Let $I_{\mathrm{t}}(\underline{D})$ and $I_{\mathrm{u}}(\underline{D})$ be the constraints, respectively, 
as the functions of $\underline{D}$. Yamaji (2015) derived the equations,

$$
\begin{aligned}
& I_{\mathrm{t}}(\underline{D})=-\log _{2}\left[\frac{1}{M} \sum_{m=1}^{M} H\left(\lambda^{(m)} \underline{D} \sigma_{4}^{(m)}-1\right)\right] \\
& I_{\mathrm{u}}(\underline{D})=-\log _{2}\left[\frac{1}{M} \sum_{m=1}^{M} H\left(1-\lambda^{(m)} \underline{D} \sigma_{4}^{(m)}\right)\right],
\end{aligned}
$$

where $\sigma_{4}^{(m)}$ is the fourth coordinate of the $m$ th of $\vec{\sigma}^{(1)}, \ldots, \vec{\sigma}^{(M)}$, which denote the uniformly distributed $M$ points on $\mathrm{S}(M \gg 1)$, and $\lambda^{(m)}$ is derived from $\vec{\sigma}^{(m)}$ through the procedure in Appendix A. Figure 2a shows the graphs of $I_{\mathrm{t}}(\underline{D})$ and $I_{\mathrm{u}}(\underline{D})$.

As a result, we use the function,

$$
F(\underline{\vec{x}})=I_{\mathrm{t}}(\underline{D}) n_{\mathrm{t}}(\underline{\overrightarrow{\vec{x}}})+I_{\mathrm{u}}(\underline{D}) n_{\mathrm{u}}(\underline{\vec{x}}),
$$

to evaluate the fitness of $\underline{\vec{x}}$ to a given data set, where $I_{\mathrm{t}}(\underline{D})$ and $I_{\mathrm{u}}(\underline{D})$ are the weights of the numbers, $n_{\mathrm{t}}(\underline{\vec{x}})$ and $n_{\mathrm{u}}(\underline{\vec{x}})$. The vector, $\underline{\vec{x}}$, that maximize this function is thought to be the optimal stress for a given data set. This scheme attempts to fit a spherical cap (Fig. 1) to the distribution of points on $S$ corresponding to twin and untwin data. Even if the feasible region does not exist, the best solution is obtained by this scheme.

\subsection{Numerical method}

The optimal $\underline{\vec{x}}$ was determined using the large number of uniformly distributed points on $\mathrm{S}$ as the computational grid in this work. An exhaustive search was employed to find the optimal point among them. The 60,000 points generated by Yamaji and Sato (2012) were used for this purpose. Let $\vec{\sigma}^{(1)}, \ldots, \vec{\sigma}^{(M)}$, be the unit vectors representing the points, where $M=60,000$. Note that $F(\underline{\vec{x}})$ can be rewritten as $F(\vec{\sigma}, \underline{D})$. So, we identify $\underline{\vec{x}}$ and the pair, $\vec{\sigma}$ and $\underline{D}$. It is easy to calculate $\lambda$ from $\vec{\sigma}$ (Appendix A). There is no need to search $\underline{D}$ smaller than 2 , because twinning does not occur at the stress magnitudes.

The optimization of $F(\underline{\vec{x}})$ was done through the procedure:

1. Choose the $m$ trial $\underline{D}$ values, $\underline{D}^{(1)}, \ldots, \underline{D}^{(m)}$, greater than 2 .

2. Repeat the calculation, $F^{(i)}=\max _{j=1}^{M} F\left(\vec{\sigma}^{(j)}, \underline{D}^{(i)}\right)$, for $i=$ $1, \ldots, m$.

3. Find the maximum among $F^{(1)}, \ldots, F^{(m)}$. Let $\underline{D}_{\mathrm{opt}}$ and $\vec{\sigma}_{\text {opt }}$ be the differential stress and the sigma-vector corresponding to the maximum.

4. Decompose $\vec{\sigma}_{\text {opt }}$ into the optimal orientations of stress axes and stress ratio (Appendix A).

5. In order to estimate the uncertainty of the optimal ones, find the sigma-vectors satisfying $F\left(\vec{\sigma}^{(j)}, \underline{D}_{\text {opt }}\right)>0.9 F_{\text {max }}$.

6. Decompose the sigma-vectors into the orientations of $\sigma_{1}$ and $\sigma_{3}$-axes and stress ratios, and plot them on paired equal-area projections.

In case the differential stress to be detected is greater than $\sim 100$ $\mathrm{MPa}, F(\underline{\vec{x}})$ can have the multiple peaks with the heights similar to the maximum, $F_{\max }=\max _{i=1}^{m} F^{(i)}$. The proximity in their height is quantitatively defined as $\left(F_{\max }-F^{(i)}\right) / F_{\max }<0.1$. Then, the $\underline{D}$ values corresponding to the peaks that satisfy this condition were averaged to give $\underline{D}_{\mathrm{opt}}$ in the third step, where the trial $\underline{D}$ values have regular intervals.

\subsection{Error propagation}

Let $\delta \Psi, \delta \lambda$ and $\delta \underline{D}$ be errors in $\Psi, \lambda$ and $\underline{D}$, respectively. Error propagation from $\bar{\delta} \Psi$ to $\lambda$ and $\underline{D}$ is calculated as follows. Differentiating $\cos \Psi=1 / \lambda D$, we have $(\partial \Psi / \partial \lambda) \sin \Psi=(\cos \Psi) / \lambda$ and $(\partial \Psi / \partial \underline{D}) \sin \Psi=(\cos \Psi) / \underline{D}$. Then, the error propagation is denoted by the equations,

$$
\begin{gathered}
\delta \lambda=(\lambda \tan \Psi) \delta \Psi \\
\delta \underline{D}=\left(\frac{\tan \Psi}{\lambda \cos \Psi}\right) \delta \Psi,
\end{gathered}
$$

where $\underline{D}=1 / \lambda \cos \Psi$ is used. It means that both $\delta \lambda$ and $\delta \underline{D}$ increase rapidly with increasing $\Psi$, especially for large $\Psi$, and that error in $\underline{D}$ is greater than that in $\lambda$ by $1.5-2$ orders of magnitude. Figure $2 \mathrm{~b}$ shows that $\delta \underline{D}$ inflates in the range, $\underline{D} \gtrsim 10$. This is consistent with Yamaji (2015) who points out that the orientations of twinned and untwinned $e$-planes loose the resolution in $\underline{D}$ if twin lamellae were formed at $\underline{D}$ greater than 5-10.

\subsection{Heterogeneous data}

\subsubsection{Processing of heterogeneous data}

If data are heterogeneous, there may be no x-vector that satisfies all the inequalities in (14) and (15). Even in this case, the vector, $\vec{x}$, corresponding to the stresses to be detected from the heterogeneous dataset satisfy many of the inequalities compared to those corresponding to erroneous stresses. Note that stress is constrained loosely even for $N=1$. Yamaji (2015, Fig. 7) illustrates such a case. Increasing number of data narrows the stresses compatible with the dataset. Given a heterogeneous data set, $F(\underline{\vec{x}})$ has local maxima at the x-vectors corresponding to the correct stresses. Accordingly, we searched for the peaks of $F(\underline{\vec{x}})$. To this end, the procedure described above was applied to a heterogeneous data set, and the $\mathrm{x}$-vector corresponding to the maximum of $F(\underline{\vec{x}})$ was regarded as an optimal solution. Then, the twin data that were explained by this solution were counted out to form the subset of data to which the procedure was applied again to detect the final optimal solution.

\subsubsection{Separability of stresses}

The present inverse method attempts to fit a spherical cap to the distribution of the points on $\mathrm{S}$ corresponding to twin data. Two or more spherical caps must be fitted to the distribution, when the data set is heterogeneous. If the intersection of the spherical caps has a large area compared to the areas of the caps, the separation is not easy (Fig. 3). It is impossible, if the larger cap includes the smaller one.

Accordingly, we propose a measure of easiness to separate stresses,

$$
\mathrm{Sp}=1-S_{\mathrm{int}} / \min \left(S^{(1)}, S^{(2)}\right),
$$

where $S^{(1)}$ and $S^{(2)}$ are the areas of the spherical caps of the two stresses to be separated, $S_{\text {int }}$ is the area of of their intersection. $S^{(1)}$ and $S^{(2)}$ were calculated from the $\Psi$ values of the caps (Appendix B), whereas $S_{\text {int }}$ was evaluated numerically. The present article has the MATLAB M-files as supplementary materials to calculate $\mathrm{Sp}$ (Appendix C). 


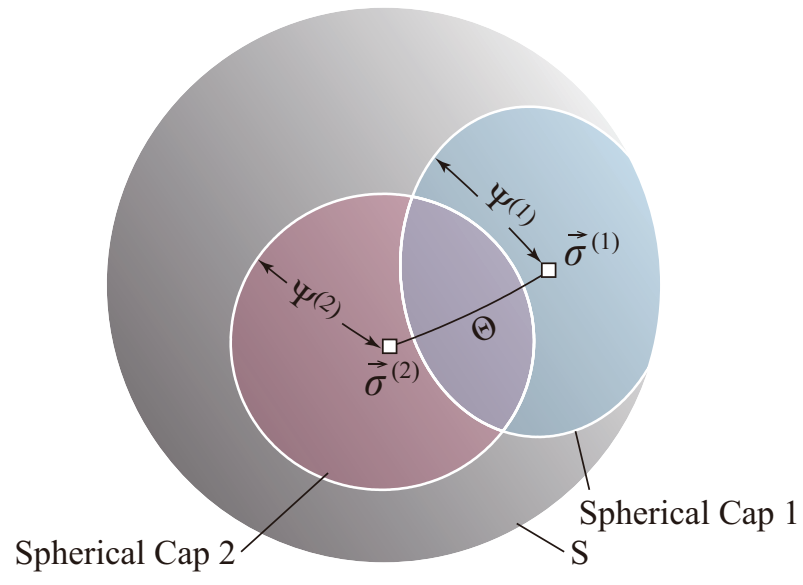

Figure 3: Schematic illustration for the explanation of the separability, the difficulty measure for the separation of stresses from heterogeneous twin data. The stresses are represented by Spherical Caps 1 and 2. The separation is easy, if they do not have an intersection. It is difficult, if the intersection is as large compared to the size of the smaller spherical cap. The separation is impossible, if the smaller cone is encompassed by the larger one. The Euclidean and angular distances between the points $\vec{\sigma}^{(1)}$ and $\vec{\sigma}^{(2)}$ on $\mathrm{S}$ are denoted, respectively, by $d_{\mathrm{OL}}$ and $\Theta$.

We call Sp the separability of stresses; and Sp has a value between 0 and 1. Spherical caps without intersection have $S p=1$, meaning that the corresponding tensors can be easily separated. The smaller this measure is, the more difficult the separation is. If the smaller cap is included by the larger one, the separation is impossible and $\mathrm{Sp}=0$.

\subsection{Dissimilarity between deviatoric stress tensors}

We define the dissimilarity, $d$, between assumed and obtained deviatoric stress tensors to estimate the accuracy of the present method. Let us distinguish the quantities related with the two tensors by the superscripts, (1) and (2). For example, the fivedimensional position vector, $T_{\mathrm{E}}^{(i)} \vec{\sigma}^{(i)}=\sqrt{3} \lambda^{(i)} D^{(i)} \tau_{\mathrm{c}} \vec{\sigma}^{(i)}$, represents the $i$ th tensor (Fqs. 3 and 6). We use the Euclidean distance between the endpoints of the vectors,

$$
d \equiv\left|T_{\mathrm{E}}^{(1)} \vec{\sigma}^{(1)}-T_{\mathrm{E}}^{(2)} \vec{\sigma}^{(2)}\right|=\sqrt{3}\left|\lambda^{(1)} D^{(1)} \vec{\sigma}^{(1)}-\lambda^{(2)} D^{(2)} \vec{\sigma}^{(2)}\right| \tau_{\mathrm{c}} .
$$

as the dissimilarity. Since $T_{\mathrm{E}}$ and $\vec{\sigma}$ are invariant under coordinate rotations in the physical space, $d$ is invariant as well. Two deviatoric stress tensors are identical, if and only if $d=0$. The dissimilarity increases with increasing differences in the principal orientations, stress ratios or deviatoric stresses. The nondimensionalized dissimilarity, $\underline{d}=d / \tau_{\mathrm{c}}$, is also useful in the following discussions.

\section{Test}

\subsection{Generation of synthetic data sets}

The present inverse method was tested with a number of synthetic data sets, which were generated as follows. Calcite aggregate was assumed to have $N_{\mathrm{g}}$ grains with isotropic lattice fabric. Since each grain has three $e$-planes, we have $N=3 N_{\mathrm{g}}$. To test
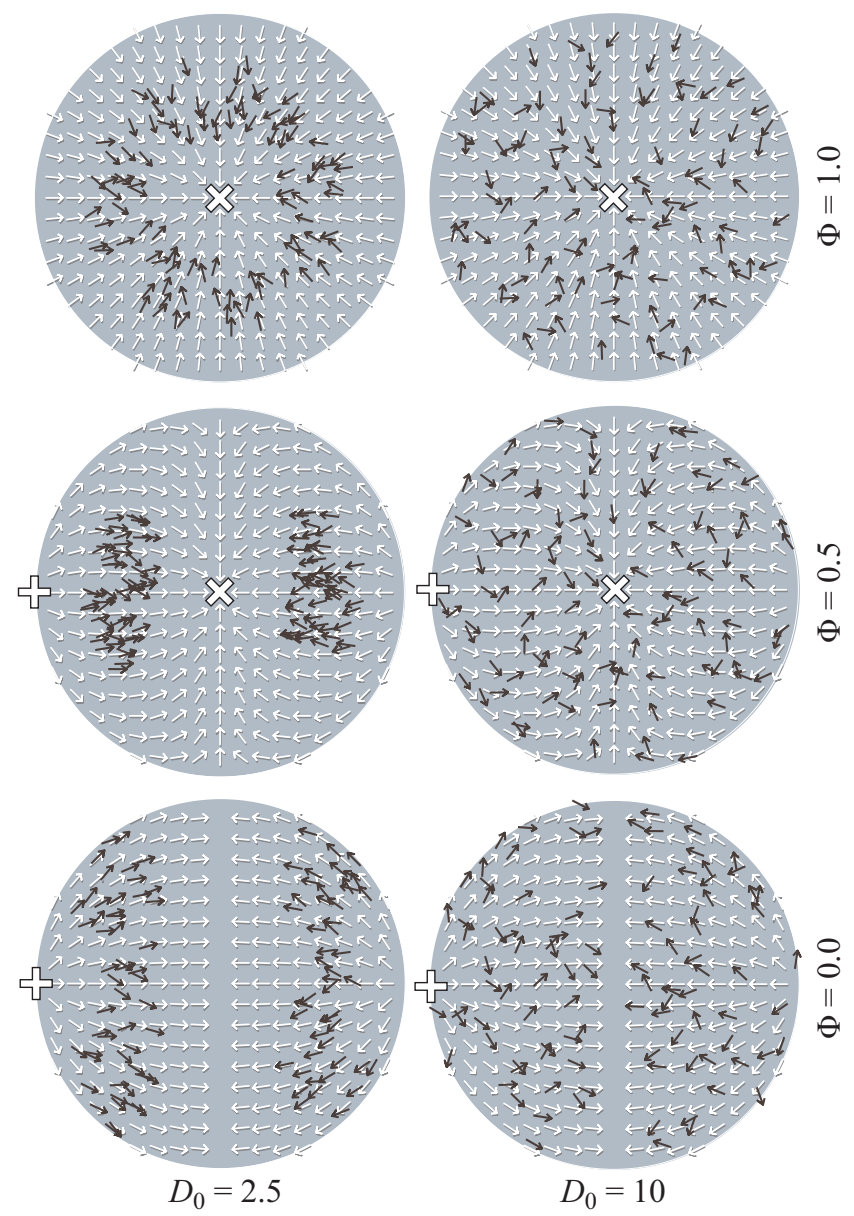

Figure 4: Tangnet-lineation diagrams (equal-area projections) showing the examples of synthetic twin data (solid arrows). The arrows indicate the gliding directions of the 'footwalls.' Bold crosses denote the assumed principal orientations: $+=\sigma_{1}$-axis, $\times=\sigma_{3}$-axis. White arrows indicate maximum shearing directions. In each diagram, 100 data are plotted. Randomly oriented untwin data are not plotted here for simplicity.

the stability of the inversion by the increasing number of data, a few different numbers in the range, 75-300, were assigned to $N_{\mathrm{g}}$. To simulate the fabric, the $c$-axes of the grains were oriented with uniform angular intervals: The uniform distribution was approximated by the generalized spiral set (Rakhamanov et al., 1994). The $a$-axes were randomly rotated about the $c$-axes. Given $\boldsymbol{c}$ and $\boldsymbol{e}$, the gliding direction on an $e$-plane was obtained by $\boldsymbol{g} \propto(\boldsymbol{I}-\boldsymbol{e e})(\boldsymbol{c}-\boldsymbol{e})$, where $(\boldsymbol{I}-\boldsymbol{e e})$ is called elementary orthogonal projector (Meyer, 2000), $\boldsymbol{e} \boldsymbol{e}$ is the square matrix whose $i j$ th component is $e_{i} e_{j}$, and the constant of proportionality is determined by the condition, $|\boldsymbol{g}|=1$.

A homogeneous data set was generated with the set of assumed stress ratio and stress axes and non-dimensional differential stress, $\underline{D}_{0}$. Given these parameters, we calculated $\boldsymbol{s}$ through Eq. (1). The twinning condition, $\tau \geq \tau_{\mathrm{c}}$, is nondimensionalized as $\lambda \underline{D} \boldsymbol{g} \cdot \boldsymbol{s} \boldsymbol{e} \geq 1$, thereby we judge whether $e$-planes satisfied the twinning condition. The examples of synthetic data sets are shown in Fig. 4.

A heterogeneous dataset from which $K$ stress states should 
(a)

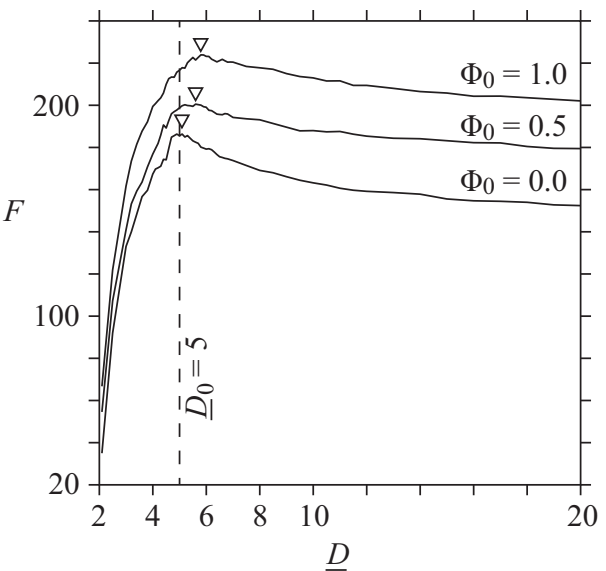

(b)

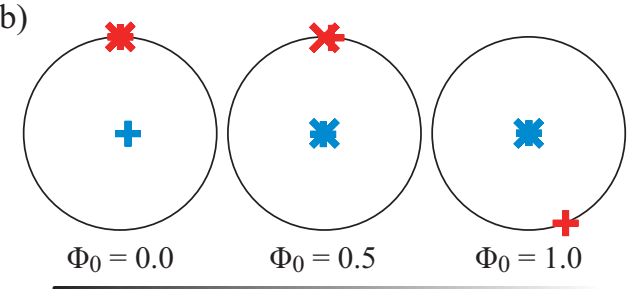

$\mathbf{X} \sigma_{1 \text {-axis (assumed) }}+\sigma_{1}$-axis (obtained) $\times \sigma_{3}$-axis (assumed) $+\sigma_{3}$-axis (obtained)

(c)

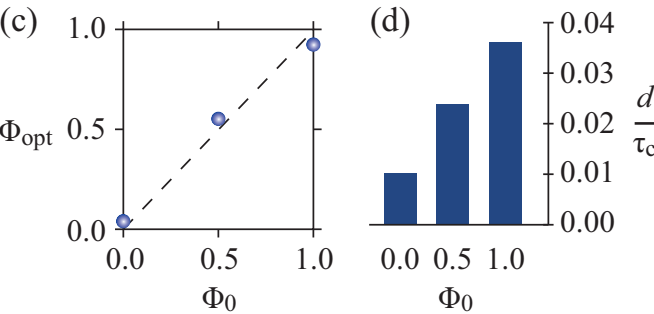

Figure 5: The results of the present method applied to the three homogeneous datasets that were generated with assuming the same differential stress except for the stress ratios, $\Phi_{0}$ at $0.0,0.5$ and 1.0. (a) $F$ versus $D$ showing the assumed (dashed line) and optimal (triangles) $D$ values. (b) Equal-area projections showing the assumed and optimal principal orientations. (c) The assumed and optimal $\Phi$ values. (d) Dissimilarities of the assumed and optimal differential stress tensors normalized by $\tau_{\mathrm{c}}$.

be separated was formed by the following procedure. First, the resolved shear stresses along each $e$-plane under the assumed $K$ stresses were calculated. We refer $\tau^{1}, \ldots, \tau^{K}$ to them. Second, if at least one of the inequalities, $\tau^{1} \geq \tau_{\mathrm{c}}, \ldots, \tau^{K} \geq \tau_{\mathrm{c}}$, is satisfied, the $e$-plane was judged to be twinned. This judgment was done for all $e$-planes to make a heterogeneous dataset.

\subsection{Homogeneous data}

The twinning condition, $\tau \geq \tau_{\mathrm{c}}$, requires $\underline{D} \geq 2$. When differential stress is low (e.g., $\underline{D}_{0}=2.5$ ), twinning occurs only along the $e$-planes that are more or less favorably oriented with respect to the stress axes and that have the gliding directions subparallel to the maximum shearing directions (Fig. 4). In addition, the poles to the twinned $e$-planes make clusters or girdles depending on the $\Phi$ value. The symmetry of the clusters reflects that of assumed deviatoric stress tensor. That is, they make small-circle girdles for $\Phi=0$ or 1 . Otherwise, they make more or less elliptical clusters with orthorhombic symmetry, because the maximum shearing directions have such symmetry. These features allow us to detect easily the assumed stresses from the twin lamellae that were formed at low a differential stress. In contrast, when differential stress is large (e.g., $\underline{D}_{0}=10$ ), those features become unclear, and many $e$-planes with unfavorably oriented $\boldsymbol{e}$ and $\boldsymbol{g}$ vectors are allowed to be twinned. As a result, it is not easy to invert the data from twin lamellae that were formed at a high differential stress.

The present inverse method was applied, first, to three homogeneous datasets $\left(N_{\mathrm{g}}=100\right)$ that were generated with the same deviatoric stress tensors $\left(\underline{D}_{0}=5.0, \mathrm{~N}-\mathrm{S}\right.$ trending $\sigma_{1}$-axis and vertical $\sigma_{3}$-axis) except for their $\Phi$ values $(0.0,0.5$ and 1.0$)$ (Fig. 5). As a result, $F$ showed the maximum at $\underline{D}_{\text {opt }}=5.1,5.6$ and 5.8. The optimal principal orientations were determined within several degrees from the assumed ones. The dataset that were generated with $\Phi_{0}=0.0$ and 1.0 had the smallest and largest differences between assumed and optimal $\Phi$ values. The dissimilarity between the assumed and optimal deviatoric stress tensors was of the order of $\tau_{\mathrm{c}} / 100$. It means that the assumed stress conditions were detected with satisfactory accuracy, because the dissimilarity was much smaller than the uncertainty of $\tau_{\mathrm{c}}$ value itself.

The dissimilarity between the assumed and optimal deviatoric stress tensors showed that the accuracy of the solutions was independent from the assumed $\Phi$ values (Fig. 5d). The same test was done with the three $\Phi_{0}$ values not only for $\underline{D}_{0}=5.0$ but also $\underline{D}_{0}=2.5$ and 7.5. As a result, the accuracy of the solutions was independent from $\Phi_{0}$ values. Accordingly, we use the value, $\Phi_{0}=0.5$, in the following tests.

A series of numerical experiments was performed using the assumed deviatoric stress tensors with common stress ratio and principal orientations but with different $\underline{D}_{0}$ values. As a result, it was found that $F(\underline{\vec{x}})$ had a clear peak when $\underline{D}_{0}$ was smaller than $\sim 3$. The graph of $F(\underline{\vec{x}})$ had multiple peaks for $\underline{D}_{0} \gtrsim 3$ (Fig. a). And, the peak became a plateau with zig-zag top for $\underline{D}_{0} \gtrsim 7$, giving rise to the numerical instability in $\underline{D}_{\mathrm{opt}}$. This means that the determination of stress magnitude was accurate only for the data sets that were generated with low differential stresses.

$\underline{D}_{\text {opt }}$ showed a significant scatter for the data sets with $\underline{D}_{0}$ greater than 50-70 MPa, but it was found that the relationship between $\underline{D}_{0}$ and $\underline{D}_{\mathrm{opt}}$ is approximated by the equation,

$$
\log _{10} \underline{D}_{0}=\log _{10} 2+\left(\underline{D}_{\mathrm{opt}}-2\right) / a
$$

where $a$ is the constant that depends on $N_{\mathrm{g}}$ (Fig. b). This equation can be used to calibrate $\underline{D}_{\text {opt }}$ with the differential stress that should be determined from a data set. The regression analyses of the data in Fig. $6 \mathrm{~b}$ yielded the values of $a$ in Eq. (22): $a=6.82$ for $N_{\mathrm{g}}=300, a=5.76$ for $N_{\mathrm{g}}=150$ and $a=4.52$ for $N_{\mathrm{g}}=75$. Although $\underline{D}_{\text {opt }}$ became unstable for $\underline{D}_{0} \gtrsim 7$, the present method accurately determined the stress ratio and stress axes from the data sets generated with large $\underline{D}_{0}$ values (Fig. 6c). 
(a)

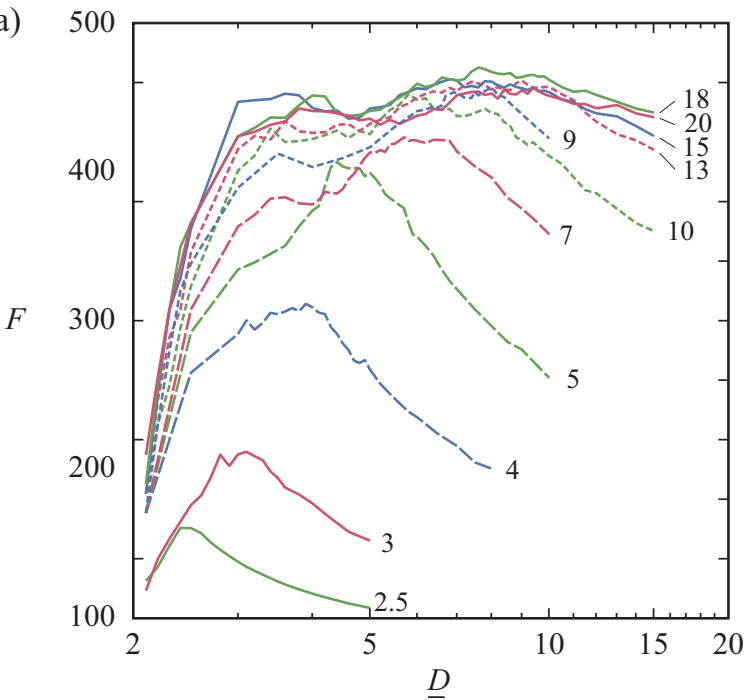

(b)

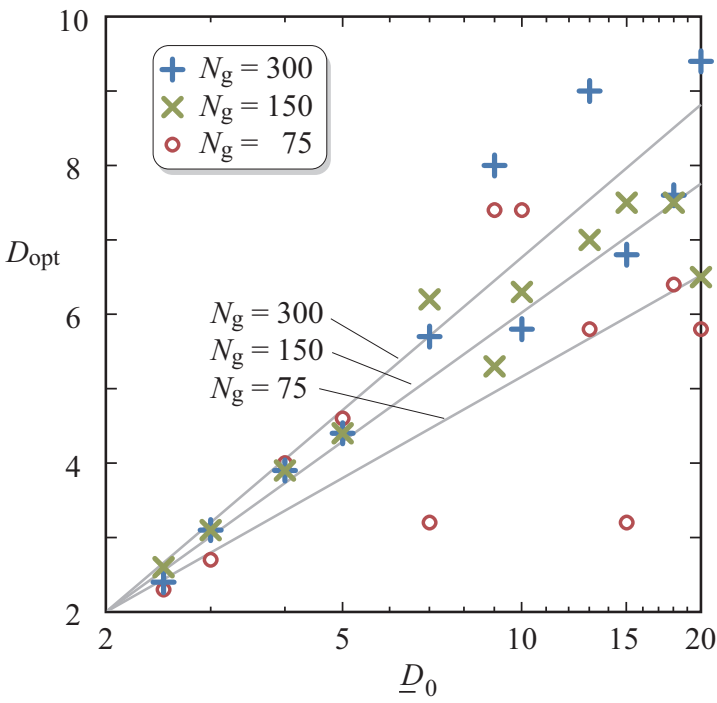

(c)
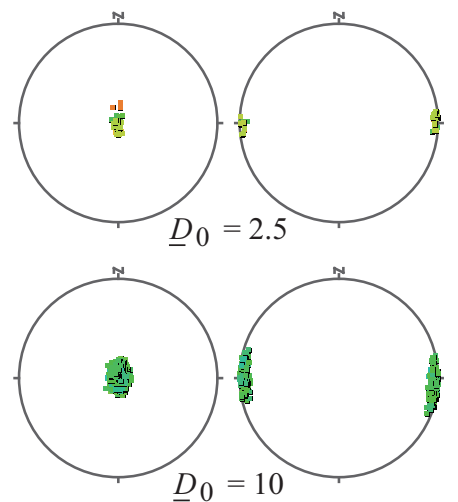
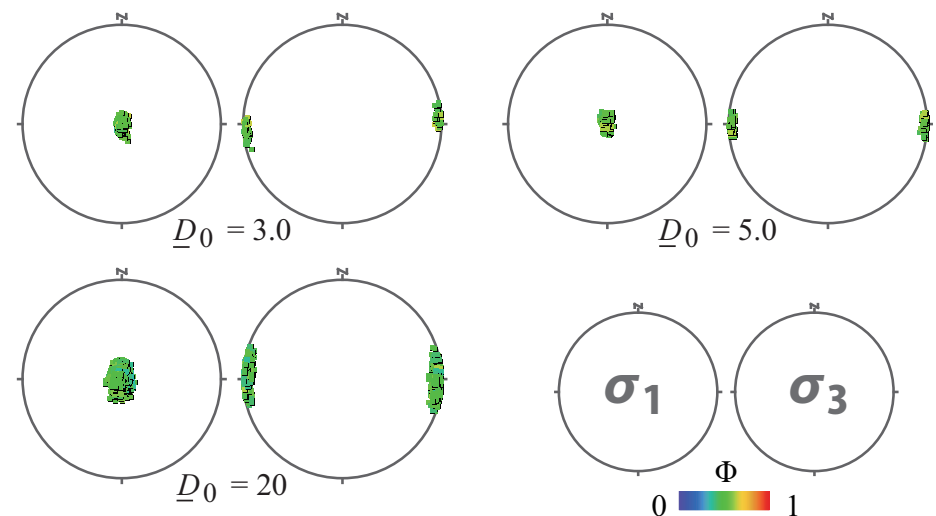

Figure 6: (a) $F$ versus $D$ for the synthetic data sets generated with the vertical $\sigma_{1}$ - and E-W trending $\sigma_{3}$-axes, $\Phi=0.5$ and $N_{\mathrm{g}}=300$. The numbers attached with lines denote the $\underline{D}_{0}$ values. (b) $\underline{D}_{\text {opt }}$ versus $\underline{D}_{0}$ for the data sets with different $N_{\mathrm{g}}$ values. Gray lines depict the regression lines (Eq. 22). (c) Paired equal-area projections showing the optimal stress axes and stress ratios determined from the data sets with $N_{\mathrm{g}}=300$. (For interpretation of the references to color in this figure legend, the reader is referred to the web version of this article.)

\subsection{Biased data}

The incorporation of untwin data is essential for stress inversion of the orientations of $e$-twin lamella to place the upper bound of differential stress. However, the sampling bias gives rise to the misclassification of twin data into untwin ones, leading possibly to erroneous results of stress inversion. The bias depends on several factors including the angle, $\chi$, between a thin section and a twin lamella (Fig. 7), width and spacing of twin lamellae, etc. Yamaji (2015) showed that at the worst case the $20-25 \%$ of twin data are misclassified into untwin ones.

Here, we test the present inverse method using a biased data set. Following Yamaji (2015), only the geometrical factor of the bias is taken into account. Twin lamellae were assumed to be observed in mutually perpendicular three thin sections. The possibility of correct classification of each twin datum was assumed to be $\sin \chi$ (Terzaghi, 1965): Twin lamellae were overlooked at the probability of $1-\sin \chi$. The rejection method (e.g., Press et al., 2007) was employed to generate a biased dataset. That is, a random number was, first, drawn from a uniform dis-

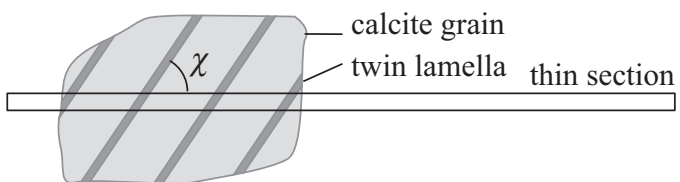

Figure 7: Schematic illustrations for the sampling bias of twin data. The twin lamellae in these pictures have the same widths and spacings, but those meeting at a low angle with a thin section tend to be overlooked under a microscope.

tribution between 0 and 1 for each twin datum. Second, if the random number was smaller than the Terzaghi factor, $\sin \chi$, of the datum, the attribute of the datum was kept as 'twin.' If, on the other hand, the random number was greater than the factor, the attribute was switched to 'untwin.' The thin section in which twins were observed was assumed to be horizontal.

As a result, 60 twin data were misclassified as 'untwinned,' and we got $N_{\mathrm{t}}=186$ and $N_{\mathrm{u}}=714$. Fig. 8a shows the Mohr diagram of the data. The symbols of twin and untwin data are 
(a)

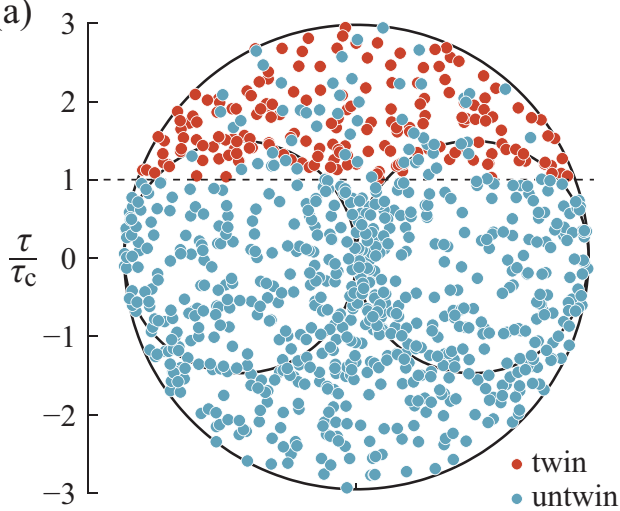

(b)

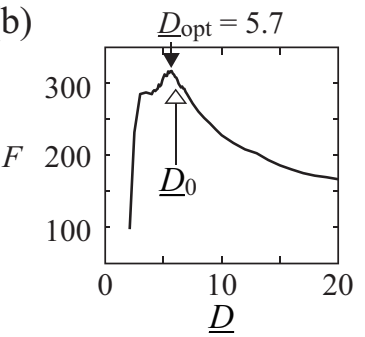

(c)

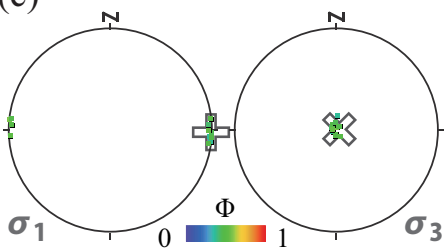

Figure 8: The effect of sampling bias. (a) Lower-hemisphere, equal-angle projection showing the synthetic data, which were generated with $N_{\mathrm{g}}=300$, $\Phi=0.5, \underline{D}_{0}=6$, and the stress axes depicted by crosses. (b) Mohr diagram showing the assumed stress for generating the data set. Sixty twin data were misclassified as untwinned ones, which are plotted above the horizontal line at $\tau / \tau_{\mathrm{c}}=1$. (c) The total score versus $\underline{D}$. (d) Paired lower-hemisphere, equal-area projections showing the optimal solution. Crosses indicate the assumed stress axes. (For interpretation of the references to color in this figure legend, the reader is referred to the web version of this article.)

mixed above the horizontal line at $\tau / \tau_{\mathrm{c}}=1$ in the diagram, indicating the misclassification. $F(\vec{x})$ showed the maximum at $\underline{D}_{\mathrm{opt}}=5.7$, which was smaller than the assumed $\underline{D}$ value by $5 \%$ (Fig. 8b). The paired equal-area projections in Fig. 8c show the principal orientations and stress ratio of the optimal stress: The assumed stress axes and stress ratio were successfully recovered. The non-dimensional differential stress calibrated by Eq. (22) was 6.98, about 1.0 greater than the assumed one.

\subsection{Variation of critical resolved shear stress}

It is known that the critical resolved shear stress, $\tau_{\mathrm{c}}$, depends weakly on a few factors including temperature and grain size. Strain hardening by $e$-twinning itself affects $\tau_{\mathrm{c}}$ as well. Lacombe (2010) summarized that the range, $\tau_{\mathrm{c}}=10 \pm 4 \mathrm{MPa}$, was appropriate for paleo stress analysis of calcite $e$-twins.

To take into account the variation of the critical value, we regarded that calcite grains have different $\tau_{\mathrm{c}}$ values, which were assumed to obey the normal distribution with the mean at 10 $\mathrm{MPa}$ and the standard deviation of 1.6 MPa (Fig. 9a). As a result, it was expected that the potential twin planes with $\tau \lesssim 6$ MPa were unlikely twinned, but those with $\tau \gtrsim 14 \mathrm{MPa}$ were twinned with a high probability, depicted by the graph of the cumulative distribution function in Fig. 10a. The sampling bias was not applied to this dataset to observe the effect of variable $\tau_{\mathrm{c}}$ values. As a result, the data set had 245 twin and 655

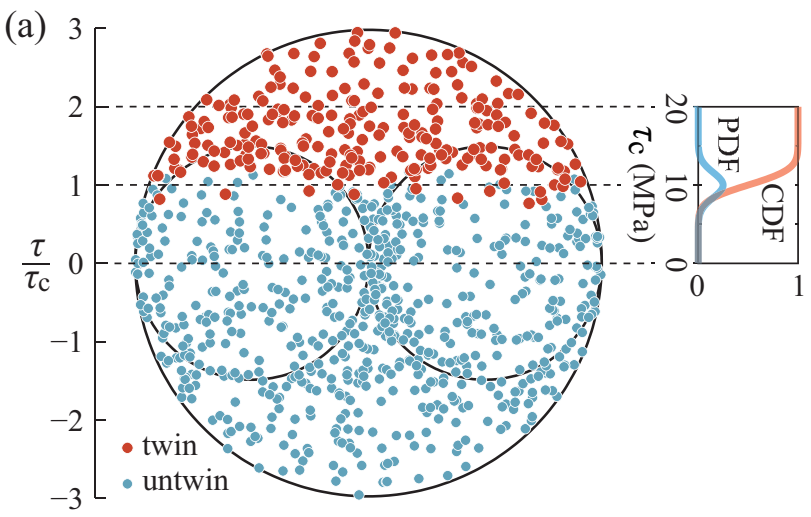

(b)
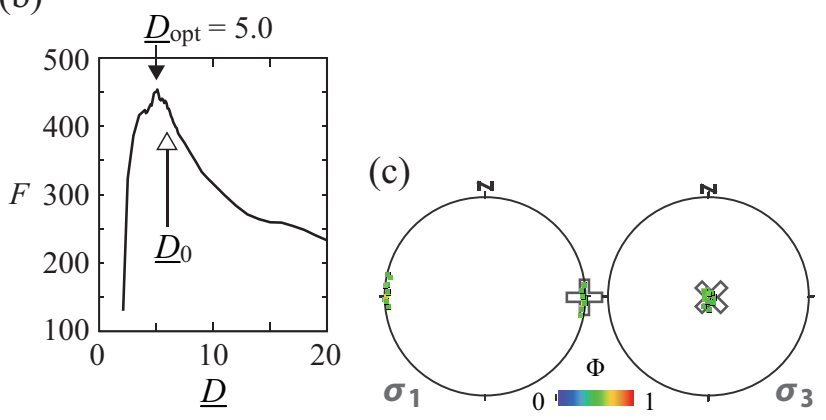

Figure 9: Effect of the variation of critical resolved shear stress. (a) The assumed probability density function (PDF) and cumulative distribution function (CDF) of $\tau_{\mathrm{c}}$. (b) Lower-hemisphere, equal-angle projection showing the synthetic data, which were generated with $N_{\mathrm{g}}=300, \Phi=0.5, \underline{D}_{0}=6$, and the stress axes depicted by crosses. (c) Mohr diagram showing the assumed stress for generating the data set. The graph 'PDF' show the probability density function of $\tau_{\mathrm{c}}$. (d) The total score versus $\underline{D}$. (e) Paired lower-hemisphere, equal-area projections showing the optimal solution. Crosses indicate the assumed stress axes. (For interpretation of the references to color in this figure legend, the reader is referred to the web version of this article.)

untwin data, among which 11 twin data and the same number of untwin data were generated with $\tau$ smaller than and greater than $10 \mathrm{MPa}$, respectively. Figure 9a shows the Mohr diagrams of the data set. The effect of variable $\tau_{\mathrm{c}}$ is clearly shown in the Mohr diagram in this subfigure: Symbols representing twin and untwin data are mingled around the horizontal line at 10 MPa. Figure $9 \mathrm{~b}$ shows the graph of $F(\vec{x})$, which has the peak at $D_{\text {opt }}=50 \mathrm{MPa}$. The differential stress calibrated by Eq. (22) is $55.1 \mathrm{MPa}$, about $5 \mathrm{MPa}$ smaller than the assumed one. The paired equal-area projections in Fig. 9c show that the assumed stress axes and stress ratio were successfully recovered by the present inverse method. As a result, we found that the variable $\tau_{\mathrm{c}}$ did not have a harmful effect on the inversion. $d=\ldots$

\subsection{Heterogeneous data}

Finally, the present method was tested with heterogeneous data sets, in particular, to see whether the method can separate stresses from them. Three stresses A, B and C (Table 12) were assumed to generate two data sets: A data set was generated with Stresses A and B, and another with Stresses A and C. The critical resolved shear stress, $\tau_{\mathrm{c}}$, was assumed to be constant; 
(a)

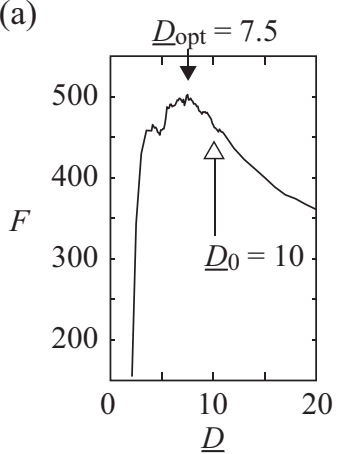

(c)

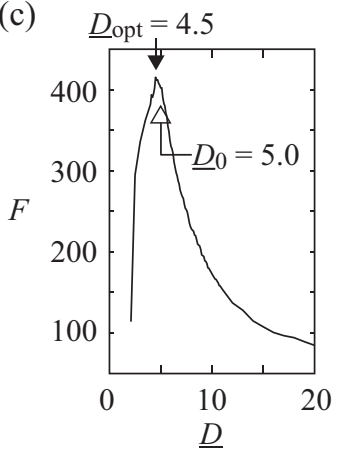

Figure 10: Homogeneous data by Stress A (a) and Stress B (b). (c) Heterogeneous data, in which an $e$-plane was judged to be twinned if it was compatible with either of the stresses. (d) The results of the present inverse method applied to the heterogeneous data set in (c). (d) $F(\vec{x})$ versus $D$ the peak at $D=75 \mathrm{MPa}$. (e) Paired equal-area projections showing the solution at $D=75 \mathrm{MPa}$. Crosses indicate the assumed stress axes. The twin data compatible with the solution were counted out to form the subset for the second processing to detect the second solution. (f) The object function for the subset showing the peak at $D=45$ MPa. (g) Paired equal-area projections showing the solution at this differential stress. (For interpretation of the references to color in this figure legend, the reader is referred to the web version of this article.)

and the sampling bias was not considered when the heterogeneous dataset was generated. The assumed 300 calcite grains were assumed to have random lattice fabric.

The spherical caps corresponding to Stresses A, B and C have the areas of about 9.26, 5.68 and 5.68, respectively. Since those of Stresses A and B do not have an intersection, we have $\mathrm{Sp}=$ 1 , indicating the separation of the stresses is easy. In contrast, the intersection of the caps of Stresses $\mathrm{A}$ and $\mathrm{C}$ has the area of $~ 3.64$. Accordingly, we have $S p \approx 0.360$, meaning that the separation of Stresses A and C is more difficult than the that of Stresses A and B.

\subsubsection{An easy case}

The present method was applied to the entire data (Fig. 11c), and obtained the satisfactorily accurate parameters of Stress A except for the underestimation of $D$ : The object function, $F(\vec{x})$, peaked at $D_{\text {opt }}=75 \mathrm{MPa}$ (Fig. 11d). The optimal solution had the $\sigma_{1}$ - and $\sigma_{3}$-axes at $003^{\circ} / 84^{\circ}$ and $270^{\circ} / 000^{\circ}$, respectively, and $\Phi=0.54$ (Fig. 11e). Since Stress A had larger differential stress than Stress B, the $\Psi$ value of Stress A was larger than that of Stress B. Accordingly, the number of twin data compatible with Stress A was significantly greater than that of Stress B.
Therefore, Stress A was detected, first, by the present method.

The 297 twin data that were explained by the detected stress were counted out to form a data subset, to which the present inverse method was applied to test whether the method could detect Stress B. Fig. 11f shows the object function as a function of $D$ for this subset. The function had a prominent peak at $D=45 \mathrm{MPa}$, smaller than the assumed $D$ value only by $5 \mathrm{MPa}$. The optimal solution at $D_{\text {opt }}=45 \mathrm{MPa}$ is shown in the paired equal-area projections in Fig. $11 \mathrm{~g}$. The solution had the $\sigma_{1}$ - and $\sigma_{3}$-axes at $094^{\circ} / 01^{\circ}$ and $194^{\circ} / 86^{\circ}$, respectively, and $\Phi=0.48$, which were close to the assumed parameters of Stress B. The calibrated differential stresses are 128.2 and $46.5 \mathrm{MPa}$.

\subsubsection{A difficult case}

The second data set is shown in Figs 12a-c. The graph of $F(\vec{x})$ had a plateau between 40 and $90 \mathrm{MPa}$, but had the maximum at $77 \mathrm{MPa}$, which was smaller than the assumed $D$ of Stress A by $23 \mathrm{MPa}$ (Fig. 12d). Except for this underestimation, the assumed stress axes and stress ratio were successfully recovered (Fig. 12e). The optimal solution had the $\sigma_{1^{-}}$and $\sigma_{3}$-axes at $003^{\circ} / 84^{\circ}$ and $270^{\circ} / 000^{\circ}$, respectively, and $\Phi=0.54$.

The 305 twin data that were explained by the detected stress were counted out to form the data subset, which was inverted to determine the second significant stress. As a result, $F(\vec{x})$ exhibited peaks between 30 and $40 \mathrm{MPa}$, and had the maximum at $D_{\text {opt }}=33 \mathrm{MPa}$. At this differential stress, the optimal stress was determined to have the $\sigma_{1^{-}}$and $\sigma_{3}$-axes at $183^{\circ} / 01^{\circ}$ and $093^{\circ} / 16^{\circ}$, and $\Phi=0.19$. Stress axes was satisfactorily accurate, but stress ratio was not. Since $D$ was underestimated by $23 \%$ when Stress A was detected, only 305 twin data were explained by the stress determined from the entire data set, which involved 403 twin data. That is, some 100 twin data were remained in the subset, and made the problem to detect Stress B difficult. The calibrated differential stresses are 137.1 and 31.0 MPa.

\subsection{Interpretation}

This is consistent with the discussion by Yamaji (2015) based on the quantification of the constraints from twin and untwin data. This is also consistent with the error propagation illustrated in Fig. 2b. Given $\underline{D}=10, \lambda=0.5$ and $\delta \Psi$ of the order of $1^{\circ} \approx 0.018$ radians, it follows from Eq. (20) that $\delta \underline{D} \approx 1$. In case of $\underline{D}=5$, we have $\delta \underline{D} \approx 2$. Therefore, if twins were formed under the differential stresses of the order of $\sim 10^{0} \tau_{\mathrm{c}}$, $\underline{D}_{\text {opt }}$ has errors of the order of $10^{-1}$. If twins were formed at hundreds of $\mathrm{MPa}$, the errors are at least of the order of $10^{1} \mathrm{MPa}$.

Consequently, the present method was robust to the sampling bias.

The mean or representative value of the variable $\tau_{\mathrm{c}}$ is determined by the inversion. In this numerical experiment, we assumed the mean and standard deviation at 10 and $1.6 \mathrm{MPa}$, respectively, to realize the range of $\tau_{\mathrm{c}}$ values that was summarized by Lacombe (2010). It means that the uncertainty of the actual $\tau_{\text {c }}$ value at $16 \%$ leads to the same percentage of the $D$ value estimated by the inversion. This uncertainty is a few points smaller than that of Lacombe (2010, p. 826) at 20\%. However, the uncertainty of $D$ comes form not only the uncertainty of 
(a)

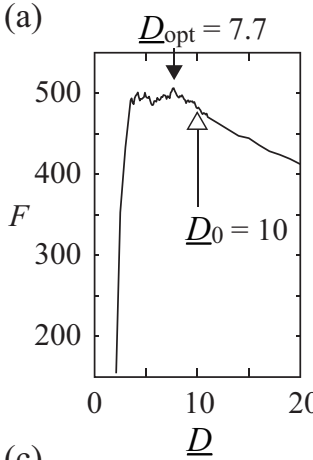

(c)

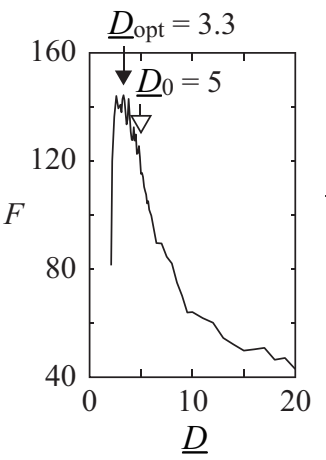

(b)

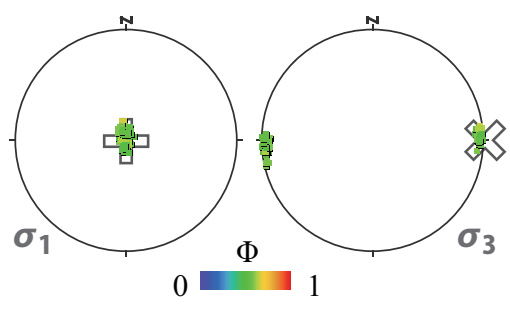

(d)

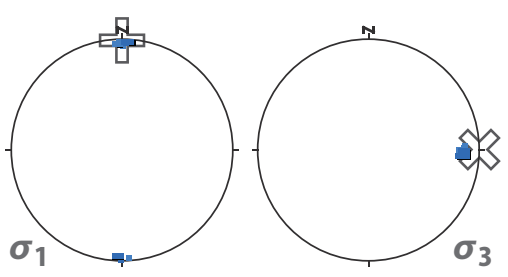

Figure 11: Homogeneous data by Stress A (a) and Stress C (b). (c) Heterogeneous data. (d) The results of the present inverse method applied to the heterogeneous data set in (c). (d) $F(\vec{x})$ versus $D$. (e) Paired lower-hemisphere, equal-area projections showing the solution at $D=77 \mathrm{MPa}$. Crosses indicate the assumed stress axes. The twin data compatible with the solution were counted out to form the subset for the final processing to detect the second solution. (f) The object function for the subset showing the peak at $D=33 \mathrm{MPa}$. (g) Paired equal-area projections showing the solution at this differential stress. (For interpretation of the references to color in this figure legend, the reader is referred to the web version of this article.)

the actual $\tau_{\mathrm{c}}$ value but also the instability of inversion schemes and the limited information contents of twin and untwin data themselves. Yamaji (2015) pointed at that the orientations of twinned and untwinned calcite e-planes lost the resolution of $D$ if twin lamellae were formed at differential stresses greater than 50-100 MPa.

However, the summation places a very loose constraint on differential stress by the following reasons. The feasible region is usually elongated along the line through the origin of the five-dimensional space (Fig. 12) unless a small spherical cap representing the solution of the inversion is fringed by many epsilon-vectors of untwin data. It follows from Eq. (6) that $|\underline{\vec{x}}|=\lambda \underline{D} \approx \underline{D} / 2$. Therefore, the resolution of $D$ depends on that of $|\underline{\vec{x}}|$, which is the distance of a point from the origin. The shape of the feasible region gives rise to the loose constraint on $\underline{D}$, if the cap is large.

The detection of axial stress ( $\Phi=0$ or 1 ) is slightly easier than that of triaxial stress with $\Phi=1 / 2$, because the area of the spherical cap corresponding to the axial stress is larger than that corresponding to triaxial stress by $\sim 10 \%$. It means that the number of $e$-planes allowed by axial stress to make twin lamellae is larger than that allowed by triaxial stress, leading to

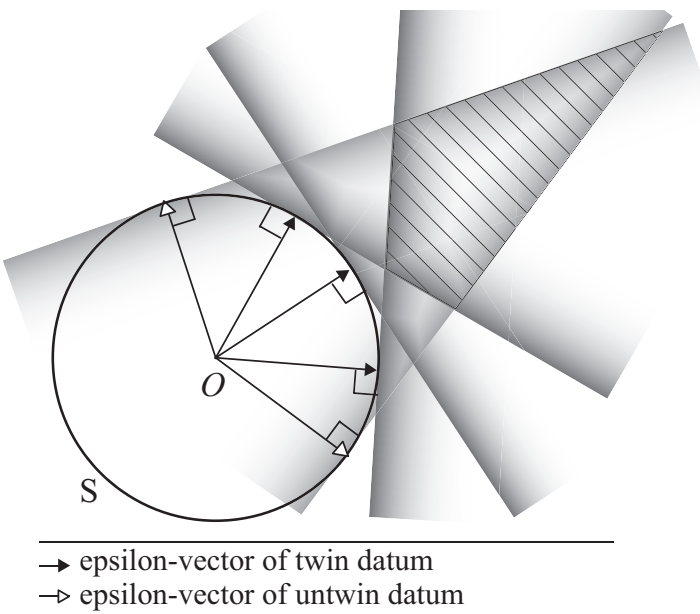

Figure 12: Schematic illustration for the feasible region (hatched area) of the linear system (Eqs. 14 and 15). Each inequality in the system limits the region on a side of the hyperplane that is tangent to $S$ and perpendicular to the epsilonvector involved in the inequality. The vector corresponding to twin and untwin data indicates the far and near sides of the hyperplane from the origin, $O$, of the space, respectively.

the difference in their detectability.

Let $D$ be the non-dimensionalized differential stress, $D=$ $\Delta \sigma / \tau_{\mathrm{c}}$, which is related to the the radius of $\Psi$ through the equation, $\cos \Psi=1 / \lambda D$. It follows from Eq. (B.1) that the area of the cap, $C$, is

$$
C=\frac{2 \pi^{2}}{3}\left[\frac{1}{(\lambda D)^{3}}-\frac{3}{\lambda D}+2\right] .
$$

Therefore, we have the ratio of the areas of the caps corresponding to axial $(\lambda=1 / \sqrt{3})$ and triaxial $(\lambda=1 / 2)$ stresses,

$$
\frac{C^{\text {axial }}}{C^{\text {triaxial }}}=\frac{3 D^{3}-3 \sqrt{3} D^{2}+3 \sqrt{3}}{2\left(D^{3}-3 D^{2}+4\right)}
$$

which is a decreasing function for $2<D$, and has vertical and horizontal asymptotes, $\lim _{D \rightarrow+2} C^{\text {axial }} / C^{\text {triaxial }}=\infty$ and $\lim _{D \rightarrow \infty} C^{\text {axial }} / C^{\text {triaxial }}=3 / 2$. Therefore, $C^{\text {axial }}$ is always larger than $C^{\text {triaxial }}$. If the same number of data points are distributed on the spherical caps that correspond to axial and triaxial stresses, the points on the former cap are sparser than those on the latter cap. It means that axial stresses are determined less precisely than triaxial stresses to some extent.

\section{Discussion}

\subsection{Difficulty measure for the separation of stresses}

The separability of stresses from calcite $e$-twin data was studied by Ga̧gała (2009) who used Orife and Lisle's (2003) stress difference, $d_{\mathrm{OL}}$, to evaluate the difficulty. The Euclidean distance between the points on $\mathrm{S}$ corresponding to the reduced stress tensors to be detected equals $d_{\mathrm{OL}}$ (Yamaji and Sato, 2006). The points are the centers of the spherical caps that stand for the deviatoric stress tensors to be detected. However, it is 
obvious from Fig. 3 that not only the distance between stresses but also the radii of the caps are indispensable for the evaluation of the difficulty. That is, if the caps have a large intersection, the separation is not easy. The definition of Sp takes indirectly into account the $D$ values of the stresses to be compared. Since the radius of a spherical cap, $\Psi$, is the monotonously increasing function of $D$ (Eq. 13) (Yamaji, 2015, Fig. 3), the spherical caps of the stresses with large $D$ values tend to have a large intersection, which reduces their separability.

It might appear that the Euclidean distance between the fivedimensional $\mathrm{x}$-vectors of stresses is a measure of the separability of the stresses. The distance is related with the $D$ values of the stresses. However, the distance is not appropriate for the separability measure. Suppose the deviatoric stress tensors, $\boldsymbol{T}$ and $k \boldsymbol{T}(k>0)$. If their differential stresses are greater than $2 \tau_{\mathrm{c}}$, they can form twin lamellae. However, the corresponding vectors, $\vec{x}$ and $k \vec{x}$, are colinear, and their spherical caps are concentric with each other. That is, the stresses are indistinguishable from the orientations of twin lamellae, even if the Euclidean distance between the points, $|k \vec{x}-\vec{x}|$, is large.

\subsection{Comparison with fault-slip analysis}

Several researchers adopted the graphical or numerical methods of fault-slip analyses to calcite $e$-twin data (Pfiffner and Burkhard, 1987; Nemcok et al., 1999; Jaya and Nishikawa, 2014). Although the techniques cannot determine differential stress, they are useful to to determine paleostresses if the $e$-twin lamellae were formed at a low differential stress.

In case we have twin lamellae formed under a low stress level, the twin data can be regarded as noisy fault-slip data. To see this, consider the misfit angle between the maximum shearing direction and the gliding direction of an $e$-plane (Fig. 13a). It can be seen from the Mohr circle in Fig. 13b that the maximum misfit angle, $\theta_{\max }$ is a function of $D$, and that $(D / 2) \sin \left(90^{\circ}-2 \theta_{\max }\right)=\tau_{\mathrm{c}}$. It follows that $\theta_{\max }=$ $(1 / 2) \cos ^{-1}(2 / D)$. The graph of this function is shown in Fig. $13 \mathrm{c}$. Indeed, $\theta_{\max }$ is as small as $\lesssim 30^{\circ}$, if differential stress is as low as $\lesssim 5 \tau_{\mathrm{c}}$. This tendency was obvious already in Fig. 4 . The Wallace-Bott hypothesis - the basis of fault-slip analysissays that the misfit angle associated with faulting must be zero. It means that the methods of fault-slip analysis are useful to detect reduced stress tensors from the twin lamellae that were formed under low stress levels.

The right-dihedra method (Angelier and Mechler, 1977), which was applied by Pfiffner and Burkhard (1987) and Nemcok et al. (1999) to calcite $e$-twins, assumes that a so-called beachball pattern depicts the possible $\sigma_{1}$ - and $\sigma_{3}$-orientations compatible with a twin datum. However, the twinning condition, $\tau \geq \tau_{\mathrm{c}}$, places a tighter constraint than the pattern The constraint from a twin lamella formed under a low stress level is tighter than the constraint from the pattern, whereas the opposite is true for a twin lamella formed under a high stress level (Yamaji, 2015, Fig. 7).

On the other hand, Jaya and Nishikawa (2014) employed the multiple inverse method (Yamaji, 2000) to estimate Pliocene stress field. Figure 14 demonstrates that the method succeeded in detecting the stresses that were assumed to generate twin (a)

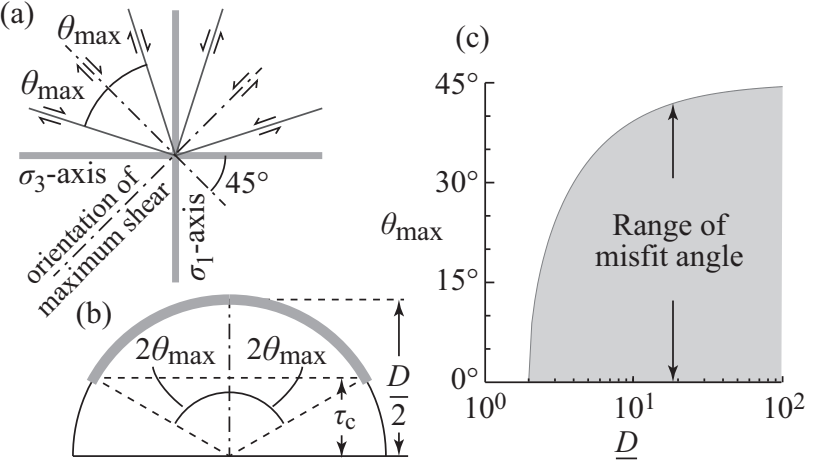

Figure 13: (a) Shematic illustration showing the range of misfit angles between the gliding and maximum shearing directions on an $e$-plane. (b) Mohr circle with the radius of $D / 2$. Thick line indicates the set of points that represents the range in (a). (c) Maximum misfit angle versus non-dimnensional deviatoric stress.

data, because the method is robust to the heterogeneity of data. However, when natural data are dealt with, the accuracy and precision of the method depends on the heterogeneity of the data. The present method was demonstrated to be robust to sampling bias and variable $\tau_{\mathrm{c}}$. However, the separation of stresses from heterogeneous $e$-twin data is not easy, more difficult than from fault-slip analysis. This difficulty comes from the tolerance. The Wallace-Bott hypothesis places a constraint

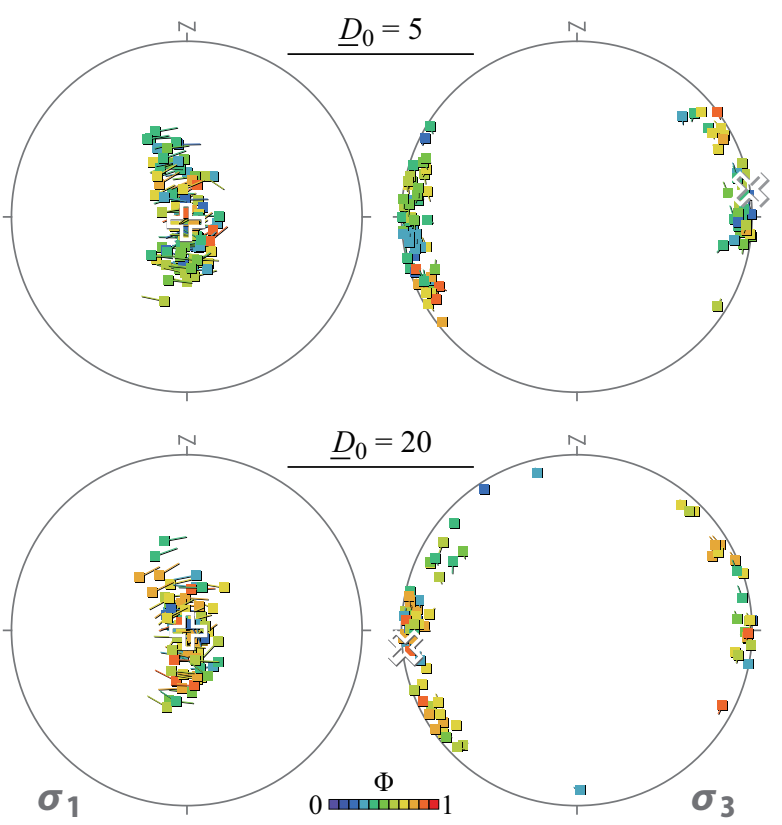

Figure 14: Multiple inverse method applied to the synthetic data of calcite $e$ twin, which were generated with $N_{\mathrm{g}}=300$ and the assumed stress, $\Phi=0.5$, vertical $\sigma_{1}$ - and E-W trending $\sigma_{3}$-axes. Crosses in these paired equal-area projections indicate the optimal stress axes determined by the method. The optimal solutions for the cases of $D_{0}=50$ and $200 \mathrm{MPa}$ had $\Phi=0.52$ and 0.51 , respectively. Lower-hemisphere, equal-area projections. (For interpretation of the references to color in this figure legend, the reader is referred to the web version of this article.) 
on stress more tightly than a twin datum.

\subsection{Laurent et al.'s (1981) inversion scheme}

Laurent et al. (1981) adopted a mathematical technique of linear programming to determine the range of deviatoric stress tensors compatible with twin and untwin data. Their method is identical with obtaining the feasible region of the inequalities (14) and (15). Given a data set free from heterogeneity and measurement errors, the region is denoted by the equation, $n(\underline{\vec{x}})=n_{\mathrm{t}}(\underline{\vec{x}})+n_{\mathrm{u}}(\underline{\vec{x}})=N$. Unfortunately, this scheme does not have good resolution in determining $\underline{D}$ (Subsection 3.1).

The feasible region exists, only if the maximum of $n(\underline{\vec{x}})$ is equal to $N$. In other case, the data set has anomaly such as heterogeneity, measurement errors, sampling bias, etc. When the solution did not exist, They proposed ad hoc to shift the hyperplanes defined by the inequalities (14) and (15) (Fig. 12). And, they determined the optimal range of deviatoric stress tensor by minimizing the sum of the shifting distances. However, such a scheme cannot cope appropriately with heterogeneous data to separate stresses, though it may be able to determine valid solutions from homogeneous but somewhat noisy data.

Unlike Laurent et al. (1981), we do not take notice of the existence of the feasible region of the inequalities (14) and (15), but regard $\underline{\vec{x}}$ at the global and local maxima of $F(\underline{\vec{x}})$ as possible solutions. In addition, not the range of $\underline{D}$ but the optimal value of $D$ is determined by our method.

It is another weakness of their scheme that it deal with twin and untwin data equally despite of their different significance on stress (Fig. 2a). Given a homogeneous data set, the epsilonvectors of twin data are confined in a hemisphere of S. So, $n_{\mathrm{u}}$ is usually larger than $n_{\mathrm{t}}$. Their solution tends to be influenced by untwin data more than twin ones.

\subsection{Etchecopar's (1984) inversion scheme}

The inversion scheme of Etchecopar (1984) is a recognized method of calcite $e$-twin stress analysis, and has been used in many areas (Lacombe, 2010, and references therein). The method has clear geometrical interpretation in terms of our parameter space, where the limitation of the method is unveiled.

In order to explain the interpretation, we utilize the nondimensional resolved shear stress along the twinning direction of an $e$-plane,

$$
\underline{\tau}=\tau / \lambda D \text {. }
$$

Note that $\underline{\tau}$ is normalized by $\lambda D$ instead of $\tau_{\mathrm{c}}$. It follows from Eqs. (9) and (23) that $\underline{\tau}=\vec{\epsilon} \cdot \vec{\sigma}=\cos \psi$, where $\vec{\epsilon}$ is the epsilonvector of the $e$-plane, and $\psi$ is the angle made by $\vec{\epsilon}$ and $\vec{\sigma}$ (Fig. 15a). Since $|\vec{\sigma}|=1, \vec{\epsilon} \cdot \vec{\sigma}$ is the length of the orthogonal projection of $\vec{\epsilon}$ onto the line parallel to $\vec{\sigma}$. If $\vec{\epsilon}$ and $\vec{\sigma}$ make an obtuse angle, $\tau$ is negative in sign (Yamaji, 2015).

Etchecopar's method attempts, first, to separate twin and untwin data plotted on a line parallel to the trial sigma-vector, $\vec{\sigma}$ (Fig. 15a). Suppose $\left\{\underline{\tau}_{\mathrm{t}}^{(1)}, \ldots, \underline{\tau}_{\mathrm{t}}^{\left(N_{\mathrm{t}}\right)}\right\}$ and $\left\{\underline{\tau}_{\mathrm{u}}^{(1)}, \ldots, \underline{\tau}_{\mathrm{u}}^{\left(N_{\mathrm{u}}\right)}\right\}$ be the sets of non-dimensional resolved shear stresses along twinned and untwinned $e$-planes, respectively. Their minimum and maximum elements are denoted as $\underline{\tau}_{\mathrm{t}}^{\min }=\min _{i=1}^{N_{\mathrm{t}}} \underline{\tau}_{\mathrm{t}}^{(i)}$ and $\underline{\tau}_{\mathrm{u}}^{\max }=\max _{i=1}^{N_{\mathrm{u}}} \underline{\tau}_{\mathrm{u}}^{(i)}$. Then, the optimal $\vec{\sigma}$ is determined by minimizing Etchecopar's object function,

$$
F_{\mathrm{E}}(\vec{\sigma})=\sum_{i=1}^{N_{\mathrm{u}}}\left(\underline{\tau}_{\mathrm{u}}^{(i)}-\underline{\tau}_{\mathrm{t}}^{\min }\right) H\left(\underline{\tau}_{\mathrm{u}}^{(i)}-\underline{\tau}_{\mathrm{t}}^{\min }\right) .
$$

The right-hand side of this equation is the summation of $\tau_{\mathrm{u}}^{(i)}-$ $\underline{\tau}_{\mathrm{t}}^{\min }$ only for the untwin data with $\underline{\tau}_{\mathrm{u}}^{(i)}>\underline{\tau}_{\mathrm{t}}^{\min }$. Given a homogeneous data free from disturbances (e.g., sampling bias, measurement errors, etc.), we have $F_{\mathrm{E}}(\vec{\sigma})=0$ at the correct $\vec{\sigma}$. Once the optimal $\vec{\sigma}$ is obtained successfully, $D$ is constrained from the condition,

$$
\underline{\tau}_{\mathrm{u}}^{\max }<\frac{\tau_{\mathrm{c}}}{\lambda D}<\underline{\tau}_{\mathrm{t}}^{\min }
$$

where $\lambda$ is calculated from the optimal $\vec{\sigma}$. It follows that

$$
\frac{\tau_{\mathrm{c}}}{\lambda \underline{\tau}_{\mathrm{u}}^{\max }}>D>\frac{\tau_{\mathrm{c}}}{\lambda \underline{\tau}_{\mathrm{t}}^{\min }}
$$

It is obvious from this equation that $F_{\mathrm{E}}(\vec{\sigma})$ takes into account only the untwin data with $\underline{\tau}$ greater than $\underline{\tau}_{t}^{\min }$. Only the epsilonvectors of untwin data in the hatched region on $\mathrm{S}$ in Fig. 15a contribute to $F_{\mathrm{E}}(\vec{\sigma})$; and remaining data are not incorporated in the function. As the trial $\vec{\sigma}$ approaches the sigma-vector to be detected, the number of such untwin data decreases and goes to zero. Therefore, only a few or one data point that have the corresponding epsilon-vectors around the margin of the spherical cap to be detected have strong influence on the optimal $\vec{\sigma}$, meaning that the inversion scheme is unstable.

The instability can make the solution of the scheme inaccurate when it deals with twin lamellae that were formed with various $\tau_{\mathrm{c}}$ values. Suppose that the values are scattered around the mean, $\bar{\tau}_{\mathrm{c}}$. Then, the epsilon-vectors corresponding to twin and untwin data are intermingled around the margin of the spherical cap to be detected (Fig. 15b). The data points of twin and untwin data are intermingled as well in the Mohr diagram in Fig. 10c. Therefore, $\underline{\tau}_{t}^{\min }$ is given by the outlier, i.e., by the twin lamellae with the minimum $\tau_{\mathrm{c}}$.

The sampling bias can spoil the optimal $D$ value, even if $\vec{\sigma}$ is correctly determined. Some of twin data are misclassified by the bias as untwin ones (Fig. 15c). The $\underline{\tau}$ values of some untwin data are intermingled with those of twin data. Tourneret and Laurent (1990) reported that such anomalies are common when actual data are processed with Etchecopar's method, though they attributed it to the heterogeneity of data and variable $\tau_{\mathrm{c}}$. In case the sampling bias is not negligible, we have

$$
\frac{\tau_{\mathrm{c}}}{\lambda D}<\underline{\tau}_{\mathrm{t}}^{\min }<\underline{\tau}_{\mathrm{u}}^{\max }
$$

instead of the inequality (24). From this condition, we can determine only the lower bound of $D$ as $D>\tau_{\mathrm{c}} / \lambda \tau_{\mathrm{t}}^{\mathrm{min}}$. It should be noted that some of untwin data are in fact twin ones, but it is unknown which untwin data are misclassified. It means that $\underline{\tau}_{t}^{\min }$ is in fact smaller than its value determined by the inversion. Accordingly, only the lower bound of $D$ is determined by Etchecopar's inverse method, if the bias is not negligible. 
(a)

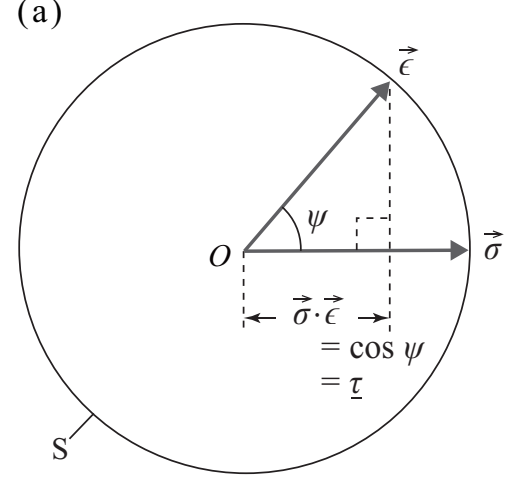

(b)

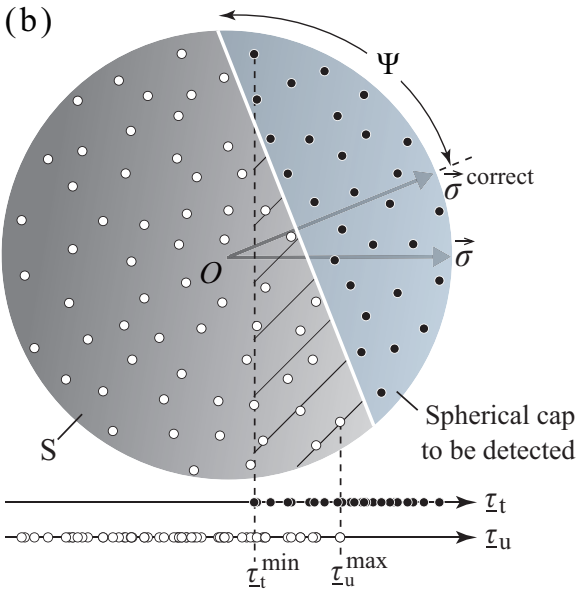

(d)

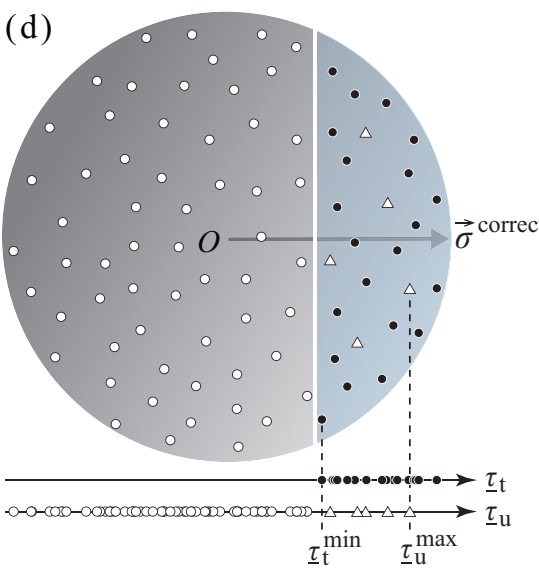

(e)

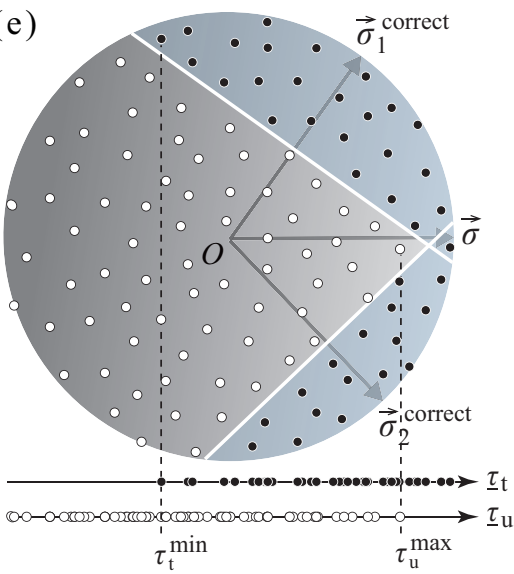

(c)

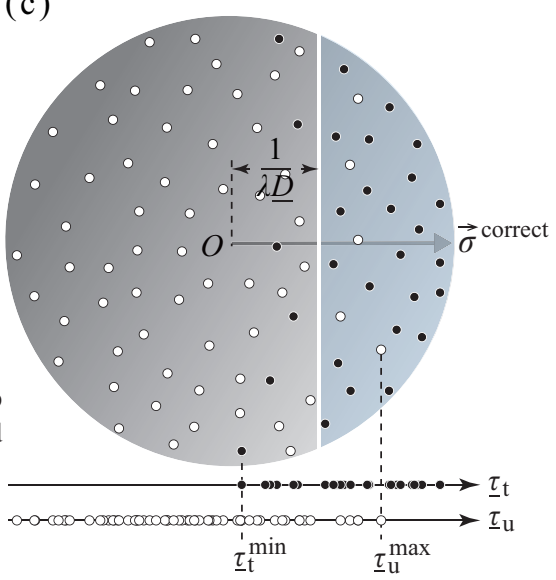

(f)

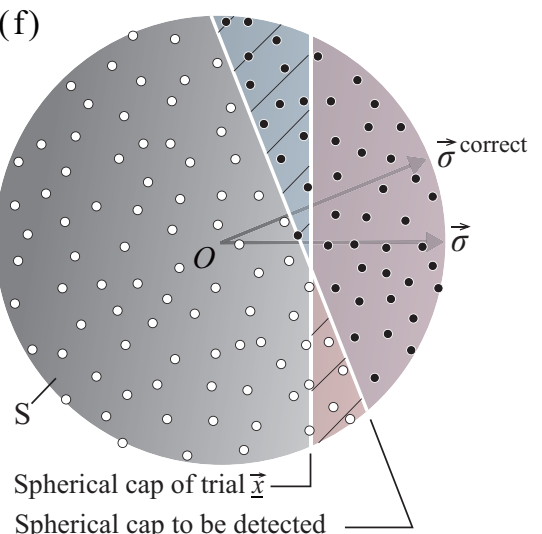

Figure 15: Schematic illustrations for the explanations of the methods of Etchecopar (1984) and Laurent et al. (1990). Solid and open circles depict the epsilonvectors corresponding to twin and untwin data, respectively. (a) The relationship of $\underline{\tau}$ and the angle between epsilon- and sigma-vectors. (b) Closed circles exist on the spherical cap centered by $\vec{\sigma}^{\text {correct }}$, which should be detected. Given a trial $\vec{\sigma}$, the orthogonal projection of the epsilon-vectors onto the line parallel to this vector yields the sets, $\left\{\underline{\tau}_{\mathrm{t}}^{(1)}, \ldots, \underline{\tau}_{\mathrm{t}}^{\left(N_{\mathrm{t}}\right)}\right\}$ and $\left\{\underline{\tau}_{\mathrm{u}}^{(1)}, \ldots, \underline{\tau}_{\mathrm{u}}^{\left(N_{\mathrm{u}}\right)}\right\}$. The minimum value among the former set is denoted by $\underline{\tau}_{\mathrm{t}}^{\text {min }}$. (c) If twin lamellae had different $\tau_{\mathrm{c}}$ values with the mean $\bar{\tau}_{\mathrm{c}}$, the epsilon-vectors corresponding to twin and untwin data are intermingled around the margin of the spherical cap. (d) The effect of sampling bias, which replaces twin data with untwin ones. Triangles depict the misclassified data. (e) Heterogeneous data-an unfavorable case for Etchecopar's inversion scheme. (f) Schematic illustration for the scheme of Laurent et al. (1990). Their object function, $F_{\mathrm{L}}(\vec{x})$ takes into account the epsilon-vectors in the hatched regions on $\mathrm{S}$. In contrast, our object function, $F(\vec{x})$ does the epsilon-vectors in the regions that are not hatched in this picture.

Even in the case of $\mathrm{Sp} \approx 0$, it would be difficult for Etchecopar's scheme to separate stresses. Fig. 15d illustrates such a case, where the spherical caps of the stresses have a small intersection. However, the elements of the set, $\left\{\underline{\tau}_{t}^{(1)}, \ldots, \underline{\tau}_{t}^{\left(N_{t}\right)}\right\}$, make a single cluster on the number line (one-dimensional space) of $\underline{\tau}$ no matter how the trial $\vec{\sigma}$ is oriented. The same is true for the elements of the set, $\left\{\underline{\tau}_{\mathrm{u}}^{(1)}, \ldots, \underline{\tau}_{\mathrm{u}}^{\left(N_{\mathrm{u}}\right)}\right\}$; and the two clusters always have an overlapping range on the number line. It is difficult to recognize the spherical caps separately in the one-dimensional space.

\subsection{Laurent et al.s' (1990) inversion scheme}

Laurent et al. (1990) regarded

$$
F_{\mathrm{L}}^{(i)}(\vec{x})=\left(\boldsymbol{\epsilon}^{(i)} \cdot \boldsymbol{x}-\tau_{\mathrm{c}}\right)^{2} H\left(\mp \boldsymbol{\epsilon}^{(i)} \cdot \boldsymbol{x} \pm \tau_{\mathrm{c}}\right)
$$

as the misfit of the trial deviatoric stress tensor for the $i$ th datum, where the upper and lower signs correspond to twin and untwin data, respectively. And, the summation, $F_{\mathrm{L}}(\vec{x})=\sum_{i=1}^{N} F_{\mathrm{L}}^{(i)}(\vec{x})$, was minimized to determine the optimal $\vec{x}$. This function indicate how much $\vec{x}$ fails to fulfill the inequalities (14) and (15). Given homogeneous data without anomaly such as sampling bias and measurement errors, this scheme works well. The epsilon-vectors that contribute to this function is confined in the hatched regions in Fig. 15e. The regions reduce and finally disappear as the spherical cap of the trial tensor approaches the spherical cap to be detected. Therefore, this inversion scheme is weak to variable $\tau_{\mathrm{c}}$.

Another problem of this scheme is that it deals with twin and untwin data equally, though they have different significance. The significance of a twin datum, $I_{\mathrm{t}}(D)$, is always greater than that of untwin datum, $I_{\mathrm{u}}(D)$. The former is about ten times great than the latter at low stress levels (Fig. 2a). As a result, the solution of Lauren et al.'s scheme is influenced by untwin data more than twin ones.

Laurent et al. (1990) noticed that natural data sets are usually 
heterogeneous. They defined a threshold value ad hoc to screen out the outliers with $F_{\mathrm{L}}^{(i)}$ greater than the threshold to detect the optimal $\vec{x}$. The multimodality of the function, $F_{\mathrm{L}}(\vec{x})$, is though to be the indication of the heterogeneity, and it is advised to use other sources of geological data to deal with heterogeneous data (Laurent et al., 1990, p. 381). For example, it may be difficult for this scheme to separate stresses in the case of Fig. 15d.

\subsection{Robustness of the present method}

Why is the present method robust to sampling bias, variable $\tau_{\mathrm{c}}$, and the heterogeneity of twin data? The method attempts to fit a spherical cap to the distribution of $\left\{\vec{\epsilon}_{\mathrm{t}}^{(1)}, \ldots, \vec{\epsilon}_{\mathrm{t}}^{\left(N_{\mathrm{t}}\right)}\right\}$ on $\mathrm{S}$, and simultaneously attempts to fit the complementary region of the cap on $\mathrm{S}$ to the distribution of $\left\{\vec{\epsilon}_{\mathrm{u}}^{(1)}, \ldots, \vec{\epsilon}_{\mathrm{u}}^{\left(N_{\mathrm{u}}\right)}\right\}$. Note that $n_{\mathrm{t}}(\vec{x})$ is the number of epsilon-vectors of twin data in the intersection of the spherical caps corresponding to the stress to be detected and to the trial stress tensor (Fig. 15e). And, $n_{\mathrm{u}}(\vec{x})$ is the number of epsilon-vectors of untwin data in the complimentary region of the union of the spherical caps. As the spherical cap of the trial deviatoric tensor approaches the spherical cap to be detected, both $n_{\mathrm{t}}(\vec{x})$ and $n_{\mathrm{u}}(\vec{x})$ in Eq. (18) increases. This is in marked contrast to the decrease of the number of data that contributes the object functions of Etchecopar (1984) and Laurent et al. (1990). The increase of $n_{\mathrm{t}}(\vec{x})$ and $n_{\mathrm{u}}(\vec{x})$ stabilizes our inversion scheme, and makes our scheme robust.

The present method is an extension of the generalized Hough transform (Ballard, 1981), which is a robust technique of image processing to detect arbitrary shapes in an image. That is, each of twin and untwin data votes stress states denoted by $\vec{x}$, and $n(\vec{x})$ stands for the number of votes. We regard the stresses that obtained majorities of votes as significant stresses. It is known that the voting with different weights improves the technique (Davies, 1987). Likewise, the votes from twin and untwin data were weighted with $I_{\mathrm{t}}(D)$ and $I_{\mathrm{u}}(D)$ in this study to improve the resolution of $D$ in Eq. (18).

The weakness of previous inversion schemes comes ultimately from the fact that they payed attention only to the onedimensional distribution of $\underline{\tau}$ (Etchecopar, 1984; Ga̧gała, 2009) or of the difference, $\tau_{\mathrm{c}}-\tau$ (Laurent et al., 1990). Since deviatoric stress tensor has five degrees of freedom, the tensor can be identified with a point in five-dimensional space. Previous inversion schemes looked at the shadows of the entities that were cast onto one-dimensional space. Suppose that two lumps of clouds at different altitudes cast shadows on the ground. Even if the shadows overlap each other, the clouds can be easily distinguished in the three-dimensional space. Separation of objects is generally easier in a high dimensional space than in a low dimensional one. Treatment of deviatoric stress tensors as five-dimensional vectors makes the separation problem easy.

In this respect, Fry (2001) was a breakthrough paper in that he showed the geometrical interpretation of stress inversion. However, his five-dimensional parameter space violates the principle of coordinate invariance (Sato and Yamaji, 2006a; Yamaji, 2015), giving rise to the difficulty of considering angles and distances in the five-dimensional space not to be affected by the choice of a coordinate system in the physical space. The ge- ometrical entities in the five-dimensional space include $|\vec{x}|$ and $\Psi$, both of which are important for the analysis of twin data.

The present method separated stresses from heterogeneous data one by one by counting out twin data compatible with previously separated stresses (Subsection 4.5). This procedure has a problem. Since a twin datum can be explained by many stresses, the counting out reduces the information contents of the data set. As a result, the second solution becomes inaccurate. There is another unsolved problem. The present method successfully detect stresses one by one, provided that the spherical caps of the stresses have different numbers of epsilonvectors. If the caps have completely the same number of the vectors, the present method detects neither of the stresses. In addition, the present and previous methods that aim at detecting stresses from heterogeneous data have a common weak point. That is, fuzzy clustering should be applied to twin data to detect the stresses by which $e$-planes were twinned. However, the method apply crisp clustering of the data, giving rise to the decreasing number of twin data and to the diminishing accuracy during the detection of the second and following solutions. This is illustrated by the inaccuracy of the second solution of the difficult case in Figs. 11c and d. It is beyond the scope of this article to solve those problems.

\section{Acknowledgments}

The author gratefully acknowledges the helpful comments by Prof. O. Lacombe and Dr. K. Amrouch to early versions of the manuscript, which improved the manuscript. Thanks are also due to Prof. T. Takeshita for his kind editorial support. This work was supported financially by JSPS (15H02141).

\section{Appendix A. Transformation from $\vec{x}$ to $T$}

Given a five-dimensional position vector, $\vec{x}$, representing a stress state, the corresponding deviatoric stress tensor, $\boldsymbol{T}$, is derived as follows. First, we obtain the sigma-vector corresponding to $\vec{x}$ as $\vec{\sigma}=\vec{x} /|\vec{x}|$. It follows from $\varsigma_{11}+\varsigma_{22}+\varsigma_{33}=0$ and Eq. (7) that

$$
\begin{gathered}
\varsigma_{11}=(\vec{\sigma})_{1}-\frac{1}{\sqrt{3}}(\vec{\sigma})_{2}, \varsigma_{22}=\frac{2}{\sqrt{3}}(\vec{\sigma})_{2}, \varsigma_{33}=-(\vec{\sigma})_{1}-\sqrt{3}(\vec{\sigma})_{2}, \\
\varsigma_{23}=\varsigma_{32}=(\vec{\sigma})_{3}, \varsigma_{31}=\varsigma_{13}=(\vec{\sigma})_{4}, \varsigma_{12}=\varsigma_{21}=(\vec{\sigma})_{5},
\end{gathered}
$$

where $(\vec{\sigma})_{i}$ is the $i$ th component of $\vec{\sigma}$. Second, solving the eigenproblem of $\boldsymbol{s}$, we have the eigenvalues, $\varsigma_{1}, \varsigma_{2}$ and $\varsigma_{3}$ $\left(\varsigma_{1} \geq \varsigma_{2} \geq \varsigma_{3}\right)$; and the corresponding eigenvectors indicate the stress axes. Stress ratio is given by $\Phi=\left(\varsigma_{2}-\varsigma_{3}\right) /\left(\varsigma_{1}-\varsigma_{3}\right)$, which yields $\lambda$ through Eq. (2). Finally, we obtain $D=|\vec{x}| / \lambda$, and $\boldsymbol{T}=\lambda D \boldsymbol{s}$.

\section{Appendix B. The area of a spherical cap on $\mathbf{S}$}

Let $\varphi_{1}, \varphi_{2}, \varphi_{3}$ and $\varphi_{4}$ be the angular coordinates on $\mathrm{S}$. Then, the area element on $S$ is $\left(\sin ^{3} \varphi_{1} \sin ^{2} \varphi_{2} \sin \varphi_{3}\right) \mathrm{d} \varphi_{1} \mathrm{~d} \varphi_{2} \mathrm{~d} \varphi_{3} \mathrm{~d} \varphi_{4}$ 
(Shakarchi, 1998, p. 351). It follows that the area of a spherical cap with the radius of $\Psi$ is

$$
\begin{aligned}
C=\int_{0}^{\Psi} \mathrm{d} \varphi_{1} \int_{0}^{\pi} \mathrm{d} \varphi_{2} \int_{0}^{\pi} \mathrm{d} \varphi_{3} \int_{0}^{2 \pi} \mathrm{d} \varphi_{4} \sin ^{3} \varphi_{1} \sin ^{2} \varphi_{2} \sin \varphi_{3} \\
=\frac{2 \pi^{2}}{3}\left(\cos ^{3} \Psi-3 \cos \Psi+2\right) .
\end{aligned}
$$

\section{Appendix C. MATLAB M-files for calculating Separabil- ity}

The following are the supplementary data related to this article: Four MATLAB M-files for calculating the separability of deviatoric stress tensors by Eq. (21). The file, 'Separability.m,' is the main program.

\section{References}

Amrouch K., Beaudoin N., Lacombe O., Bellahsen N., Daniel J.M., 2011. Paleostress magnitudes in folded sedimentary rocks. Geophysical Research Letters 38, L17301.

Amrouch K., Lacombe O., Bellahsen N., Daniel J.-M., Callot J.P., 2010, Stress/strain patterns, kinematics and deformation mechanisms in a basement-cored anticline: Sheep Mountain anticline (Wyoming, USA). Tectonics, 29, TC1005.

Angelier, J., Mechler, F., 1977. Sur une mèthode graphique de recherche des contraintes principales également utilisable en tectonique et en séismologie: La méthode des dièdres droits. Bulletin de la Socété Géologique de France 19, 1309-1318.

Beaudoin, N., Lepretre, R., Bellahsen, N., Lacombe, O., Amrouch, K., Callot, J.-P., Emmanuel, L., Daniel, J.-M., 2012. Structural and microstructural evolution of the Rattlesnake Mountain Anticline (Wyoming, USA): New insights into the Sevier and Laramide orogenic stress build-up in the Bighorn Basin. Tectonophysics 576-577, 20-45.

Bullard, D.H., 1981. Generalizing the Hough transform to detect arbitrary shapes. Pattern Recognition 13, 111-122.

Burkhard, M., 1993. Calcite twins, their geometry, appearance and significance as stress-strain markers and indicators of tectonic regime: A review. Journal of Structural Geology 15, 351-368.

Davies, E.R., 1987. A new framework for analysing the properties of the generalized Hough transform. Pettern Recognition Letters 6, 1-7.

Etchecopar, A., 1984. Etude des états de contrainte en tectonique cassante et simulation de déformations plastiques (approche mathématique). $\mathrm{PhD}$ Thesis, Univ. Montpellier.

Fry, N., 2001. Stress space: Striated faults, deformation twins, and their constraints on paleostress. Journal of Structural Geology 23, 1-9.

Ga̧gała, Ł., 2009. Reliability of selected procedures of stress inversion and data separation for inhomogeneous populations of calcite twins and striated faults: Insights from numerical experiments. International Journal of Earth Science 98, 461-479.

Hill, R., 1950. The Mathematical Theory of Plasticity. Clarendon Press, Oxford.

Jaya, A., Nishikawa, O., 2014. Paleostress reconstruction from calcite twin and fault-slip data using the multiple inverse method in the East Walanae fault zone: Implications for the Neogene contraction in South Sulawesi, Indonesia. Journal of Structural Geology 55, 34-49.

Lacombe, O., 2010. Calcite twins, a tool for tectonic studies in thrust belts and stable orogenic forelands. Oil \& Gas Science 65, 809-838.

Laurent, P., Bernard, P., Vasseur, G., Etchecopar, A., 1981. Stress tensor determination from the study of e twins in calcite: A linear programming method. Tectonophysics 78, 651-660.

Laurent, P., Tourneret, C., Laborde, O., 1990. Determining deviatoric stress tensors from calcite twins: Applications to monophased synthetic and natural polycrystals. Tectonics $9,379-389$.

Meyer, C.D., 2000. Matrix Analysis and Applied Linear Algebra. SIAM, Philadelphia.
Nemcok, M., Kovác, D., Lisle, R.J., 1999. A stress inversion procedure for polyphase calcite twin and fault/slip data sets. Journal of Structural Geology 21, 597-611.

Orife, T., Lisle, R.J., 2003. Numerical processing of paleostress results. Journal of Structural Geology 25, 949-957.

Ottosen, N.S., Ristinmaa, M., 2005. The Mechanics of Constitutive modeling. Elsevier, Amsterdam.

Pfiffner, O. A., Burkhard, M. 1987. Determination of paleo-stress axes orientations from fault, twin and earthquake data. Annales Tectonicae 1, 48-57.

Press, W.H., Teukolsky, S.A., Vetterling, W.T., Flannery, B.P., 2007. Numerical Recipes: The art of scientific computing, 3rd Edition. Cambridge University Press, Cambridge.

Rakhamanov, D.E.A., Saff, E.B., Zhou, Y.M., 1994. Minimal discrete energy on the sphere. Mathematical Research Letters 1, 647-662.

Sato, K., Yamaji, A., 2006a. Embedding stress difference in parameter space for stress tensor inversion. Journal of Structural Geology 28, 957-971.

Sato, K., Yamaji, A., 2006b. Uniform distribution of points on a hypersphere for improving the resolution of stress tensor inversion. Journal of Structural Geology 28, 972-979.

Shakarchi, R., 1998. Problems and Solutions for Undergraduate Analysis. Springer-Verlag, New York.

Takeshita, T., Tomé, C., Wenk, H.-R., Kocks, U.F., 1987. Single-crystal yield surface for trigonal lattices: Application to texture transitions in calcite polycrystals. Journal of Geophysical Resarch 92, 12917-12930.

Terzaghi, R.D., 1965. Source of error in joint surveys. Geotéchnique 15, 287304

Tourneret, C., Laurent, P., 1990. Paleo-stress orientations from calcite twins in the North Pyrenean foreland, determined by the Etchecopar inverse method. Tectonophysics 180, 287-302.

Yamaji, A., 2000. The multiple inverse method: A new technique to separate stresses from heterogeneous fault-slip data. Journal of Structural Geology 22, 441-452.

Yamaji, A., 2007. An Introduction to Tectonophysics: Theoretical Aspects of Structural Geology. Terrapub, Tokyo.

Yamaji, A., 2015. How tightly does calcite $e$-twin constrain stress? Journal of Structural Geology, in press.

Yamaji, A., Sato, K., 2006. Distances for the solutions of stress tensor inversion in relation to misfit angles that accompany the solutions. Geophysical Journal International 167, 933-942.

Yamaji, A., Sato, K., 2012. A spherical code and stress tensor inversion. Computers \& Geosciences 38, 164-167. 